\title{
Mechanism underlying the DNA-binding preferences of the Vibrio cholerae and vibriophage VP882 VqmA quorum-sensing receptors
}

\author{
Olivia P. Duddy $\circledast^{1 \odot}$, Xiuliang Huang $\oplus^{1,2 \odot}$, Justin E. Silpe $\oplus^{1 a}$, Bonnie L. Bassler $\oplus^{1,2 *}$ \\ 1 Department of Molecular Biology, Princeton University, Princeton, New Jersey, United States of America, \\ 2 Howard Hughes Medical Institute, Chevy Chase, Maryland, United States of America \\ ه These authors contributed equally to this work. \\ a Current address: Department of Chemistry and Chemical Biology, Harvard University, Cambridge, \\ Massachusetts, United States of America \\ *bbassler@princeton.edu
}

\section{G openaccess}

Citation: Duddy OP, Huang X, Silpe JE, Bassler BL (2021) Mechanism underlying the DNA-binding preferences of the Vibrio cholerae and vibriophage VP882 VqmA quorum-sensing receptors. PLoS Genet 17(7): e1009550. https://doi.org/10.1371/ journal.pgen. 1009550

Editor: Sean Crosson, Michigan State University, UNITED STATES

Received: April 10, 2021

Accepted: June 16, 2021

Published: July 6, 2021

Peer Review History: PLOS recognizes the benefits of transparency in the peer review process; therefore, we enable the publication of all of the content of peer review and author responses alongside final, published articles. The editorial history of this article is available here: https://doi.org/10.1371/journal.pgen.1009550

Copyright: @ 2021 Duddy et al. This is an open access article distributed under the terms of the Creative Commons Attribution License, which permits unrestricted use, distribution, and reproduction in any medium, provided the original author and source are credited.

Data Availability Statement: All relevant data are within the manuscript and its Supporting

Information files.

\section{Abstract}

Quorum sensing is a chemical communication process that bacteria use to coordinate group behaviors. In the global pathogen Vibrio cholerae, one quorum-sensing receptor and transcription factor, called $\mathrm{VqmA}\left(\mathrm{VqmA}_{\mathrm{Vc}}\right)$, activates expression of the $v q m R$ gene encoding the small regulatory RNA VqmR, which represses genes involved in virulence and biofilm formation. Vibriophage VP882 encodes a VqmA homolog called VqmA $A_{\text {phage }}$ that activates transcription of the phage gene qtip, and Qtip launches the phage lytic program. Curiously, $V_{q m A} A_{\text {phage }}$ can activate $v q m R$ expression but $V_{q m A_{V_{c}}}$ cannot activate expression of qtip. Here, we investigate the mechanism underlying this asymmetry. We find that promoter selectivity is driven by each VqmA DNA-binding domain and key DNA sequences in the vqmR and qtip promoters are required to maintain specificity. A protein sequenceguided mutagenesis approach revealed that the residue E194 of VqmA $A_{\text {Phage }}$ and A192, the equivalent residue in $\mathrm{VqmA}_{\mathrm{Vc}}$, in the helix-turn-helix motifs contribute to promoter-binding specificity. A genetic screen to identify VqmA $A_{\text {phage }}$ mutants that are incapable of binding the qtip promoter but maintain binding to the $v q m R$ promoter delivered additional $V q m A_{\text {phage }}$ residues located immediately $C$-terminal to the helix-turn-helix motif as required for binding the qtip promoter. Surprisingly, these residues are conserved between $V_{q m A_{P h a g e}}$ and $V_{q m A_{V c}}$. A second, targeted genetic screen revealed a region located in the $V_{q m A_{V c}}$ DNAbinding domain that is necessary to prevent $V_{q m A_{V c}}$ from binding the qtip promoter, thus restricting DNA binding to the $v q m R$ promoter. We propose that the $V q m A_{V_{c}}$ helix-turn-helix motif and the $\mathrm{C}$-terminal flanking residues function together to prohibit $\mathrm{VqmA}_{\mathrm{V}_{\mathrm{c}}}$ from binding the qtip promoter. 
Funding: This work was supported by the Howard Hughes Medical Institute, National Institutes of Health Grant R37GM065859, and National Science Foundation Grant MCB-1713731 (BLB), NIGMS T32GM007388 (OPD), a Charlotte Elizabeth Procter Fellowship provided by Princeton University, and a National Defense Science and Engineering Graduate Fellowship supported by the Department of Defense (JES). The content is solely the responsibility of the authors and does not necessarily represent the official views of the National Institutes of Health. The funders had no role in study design, data collection and analysis, decision to publish, or preparation of the manuscript.

Competing interests: The authors have declared that no competing interests exist.

\section{Author summary}

Bacteria use a chemical communication process called quorum sensing (QS) to orchestrate collective behaviors. Recent studies demonstrate that bacteria-infecting viruses, called phages, also employ chemical communication to regulate collective activities. Phages can encode virus-specific QS-like systems, or they can harbor genes encoding QS components resembling those of bacteria. The latter arrangement suggests the potential for chemical communication across domains, i.e., between bacteria and phages. Ramifications stemming from such cross-domain communication are not understood. Phage VP882 infects the global pathogen Vibrio cholerae, and "eavesdrops" on V. cholerae QS to optimize the timing of its transition from existing as a parasite to killing the host, and moreover, to manipulate $V$. cholerae biology. To accomplish these feats, phage VP882 relies on $\mathrm{VqmA}_{\text {Phage, }}$, the phage-encoded homolog of the $V$. cholerae $\mathrm{VqmA}_{\mathrm{Vc}} \mathrm{QS}$ receptor and transcription factor. $\mathrm{VqmA}_{\mathrm{Vc}}$, by contrast, is constrained to the control of only $V$. cholerae genes and is incapable of regulating phage biology. Here, we discover the molecular mechanism underpinning the asymmetric transcriptional preferences of the phage-encoded and bacteria-encoded VqmA proteins. We demonstrate how VqmA transcriptional regulation is crucial to the survival and persistence of both the pathogen $V$. cholerae, and the phage that preys on it.

\section{Introduction}

Quorum sensing (QS) is a cell-cell communication process that allows bacteria to coordinate collective behaviors [1]. QS relies on the production, release, and group-wide detection of extracellular signaling molecules called autoinducers (AIs). In the global pathogen Vibrio cholerae, the AI, 3,5-dimethyl-pyrazin-2-ol (DPO), together with its partner cytoplasmic QS receptor and transcription factor, $\mathrm{VqmA}\left(\mathrm{VqmA}_{\mathrm{Vc}}\right)$, comprises one of the $\mathrm{QS}$ circuits that controls group behaviors [2-4]. $\mathrm{VqmA}_{\mathrm{Vc}}$, following binding to DPO, activates transcription of the $v q m R$ gene encoding the small RNA, VqmR, which, in turn, represses the expression of genes required for biofilm formation and virulence factor production [2-4].

Recently, bacteria-specific viruses, called phages, have been shown to engage in densitydependent regulation of their lysis-lysogeny decisions via chemical dialogs [5,6]. Germane to our studies are phages that encode proteins resembling bacterial QS components [5,7]. Vibriophage VP882 is one such phage: It encodes the QS receptor VqmA (VqmA $\mathrm{Phage}_{\text {e }}$ ), a homolog of the $V$. cholerae $\mathrm{QS}$ receptor $\mathrm{VqmA}_{\mathrm{Vc}}$ [5]. VqmA $A_{\text {Phage, }}$ like $\mathrm{VqmA}_{\mathrm{Vc}}$, binds hostproduced DPO. DPO-bound VqmA $A_{\text {Phage }}$ activates transcription of the phage gene qtip. Qtip is an antirepressor that sequesters the phage VP882 repressor of lysis, leading to derepression of the phage lytic program and killing of the Vibrio host at high cell density $[5,8]$. Thus, the DPO AI mediates both bacterial and phage lifestyle decisions. Curiously, $\mathrm{VqmA}_{\text {Phage }}$ can substitute for $\mathrm{VqmA}_{\mathrm{Vc}}$ to activate the $V$. cholerae $v q m R$ promoter $(\mathrm{P} v q m R)$ [5]. In contrast, $\mathrm{VqmA}_{\mathrm{Vc}}$ cannot substitute for $\mathrm{VqmA}_{\text {Phage }}$ and recognize the phage VP882 qtip promoter (Pqtip). Presumably, the ability of $\mathrm{VqmA}_{\text {Phage }}$ to bind both $\mathrm{P} v q m R$ and Pqtip provides phage VP882 the capacity to influence host QS and simultaneously enact its own lysis-lysogeny decision.

$\mathrm{VqmA}_{\text {Phage }}$ shares $\sim 43 \%$ amino acid sequence identity with $\mathrm{VqmA}_{\mathrm{Vc}}$, and most of the key residues required for ligand and DNA binding are conserved [5,9]. Thus, how $\mathrm{VqmA} \mathrm{A}_{\text {Phage }}$ can recognize two different promoters, while $\mathrm{VqmA}_{\mathrm{Vc}}$ cannot, is not understood. Here, we define the mechanism underlying this asymmetry. We show that VqmA selectivity for target promoters is driven by the DNA-binding domain (DBD) of the respective protein. We identify 6 key 
nucleotides within $\mathrm{P} v q m R$ and $\mathrm{P} q t i p$ that contribute to VqmA promoter-binding selectivity, as exchanging these critical DNA sequences inverts the DNA-binding preferences of the two $\mathrm{VqmA}$ proteins. The $192^{\text {nd }}$ and $194^{\text {th }}$ residues in $\mathrm{VqmA}_{\mathrm{Vc}}$ and $\mathrm{VqmA}_{\text {Phage, }}$, respectively, within the helix-turn-helix (HTH) motifs, contribute to promoter-binding specificity. Isolation of $V_{\mathrm{Vqm}} \mathrm{A}_{\text {Phage }}$ mutants capable of activating $v q m R$ expression but incapable of activating qtip expression revealed conserved or functionally conserved residues in $\mathrm{VqmA}_{\mathrm{Phage}}$ and $\mathrm{VqmA}_{\mathrm{Vc}}$, indicating that $\mathrm{VqmA}_{\mathrm{Vc}}$ likely possesses an additional feature that prevents it from binding Pqtip DNA. A mosaic $\mathrm{VqmA}_{\mathrm{Vc}}$ protein containing the $\mathrm{VqmA}_{\text {Phage }} \mathrm{HTH}$ motif along with the C-terminal 25 flanking $\mathrm{VqmA}_{\text {Phage }}$ residues was capable of binding Pqtip. Thus, the two corresponding regions in $\mathrm{VqmA}_{\mathrm{Vc}}$ must function in concert to prevent $\mathrm{VqmA}_{\mathrm{Vc}}$ from binding to Pqtip. Together, our analyses demonstrate how VqmA $A_{\text {Phage, }}$ via its promiscuous DNA-binding activity, can control phage VP882 functions and drive host $V$. cholerae QS. Moreover, we discover why $V$. cholerae $\mathrm{VqmA}_{\mathrm{Vc}}$ cannot do the reverse, as its DNA binding is strictly constrained to the host $V$. cholerae genome.

\section{Results}

\section{VqmA promoter-binding selectivity is conferred by the DNA-binding domain}

VqmA proteins are composed of N-terminal Per-Arnt-Sim (PAS) domains responsible for binding the DPO AI and C-terminal DBDs containing HTH motifs [10]. Both VqmA $\mathrm{V}_{\mathrm{Vc}}$ and $\mathrm{VqmA}_{\text {Phage }}$ bind DPO. By contrast, with respect to DNA binding, $\mathrm{VqmA}_{\text {Phage }}$ binds to Pqtip and $\mathrm{P} v q m R$, whereas $\mathrm{VqmA}_{\mathrm{Vc}}$ only binds to $\mathrm{P} v q m R$ [5]. We reasoned that this asymmetric DNA-binding pattern arises from differences in the DBDs (S1 Fig). To test this idea, we constructed chimeras in which we exchanged the $\mathrm{VqmA}_{\mathrm{Vc}}$ and $\mathrm{VqmA}_{\text {Phage }} \mathrm{C}$-terminal domains to produce ${ }_{\mathrm{Vc}} \mathrm{N}-\mathrm{C}_{\text {Phage }}$ and ${ }_{\text {Phage }} \mathrm{N}-\mathrm{C}_{\mathrm{Vc}}$ proteins. We chose to make the junction at a residue near the C-terminal end of the PAS domain immediately following an amino acid stretch (GTIF) that is identical in both $\mathrm{VqmA}_{\mathrm{Vc}}$ and $\mathrm{VqmA}_{\text {Phage }}(\mathrm{S} 1 \mathrm{Fig})$. We cloned $v q m A_{V c}, v q m A_{\text {Phage, }}$ ${ }_{{ }_{c}} \mathrm{~N}-\mathrm{C}_{\text {Phage }}$, and ${ }_{\text {Phage }} \mathrm{N}-\mathrm{C}_{V c}$ under an arabinose-inducible promoter and transformed each construct into recombinant $\Delta t d h E$. coli harboring a $\mathrm{P} v q m R$-lux or a Pqtip-lux reporter. The Tdh enzyme is required for DPO biosynthesis, therefore a $\Delta t d h$ E. coli strain makes no DPO [3]. Apo-VqmA displays basal transcriptional activity in vivo [9]. Thus, while DPO enhances VqmA DNA-binding activity, it is not an absolute requirement for binding. Using $\Delta t d h E$. coli for these studies ensured that any transcriptional activity that occurred was exclusively a consequence of the DNA-binding capabilities of the chimeras and not ligand-binding-driven transcriptional activation of the chimeras. Consistent with our hypothesis, promoter activation by each chimera was determined by the protein from which the DBD originated: All four versions of $\mathrm{VqmA}$ activated $\mathrm{P} v q m R$-lux, whereas only $\mathrm{VqmA}_{\text {Phage }}$ and ${ }_{\mathrm{Vc}} \mathrm{N}-\mathrm{C}_{\text {Phage }}$ activated Pqtip-lux (Fig $1 \mathrm{~A}$ and $1 \mathrm{~B}$, respectively). Next, we conjugated the four versions of VqmA into $\Delta t d h$ $\Delta v q m A_{V c} V$. cholerae lysogenized by a phage VP882 mutant in which the endogenous $v q m A_{\text {Phage }}$ was inactive (VP882 vqmA $A_{\text {Phage: }}:$ Tn5). Thus, the only source of VqmA protein was that made from the plasmid. As expected, following arabinose-induction, only $\mathrm{VqmA} \mathrm{A}_{\text {Phage }}$ and ${ }_{\mathrm{Vc}} \mathrm{N}-\mathrm{C}_{\text {Phage }}$ activated qtip expression and induced host-cell lysis (Fig 1C).

We verified the above findings in vitro using electrophoretic mobility shift assays (EMSAs). Consistent with the cell-based assays, the purified $\mathrm{VqmA}_{V \mathrm{Vc}}, \mathrm{VqmA}_{\text {Phage, }} \mathrm{V}_{\mathrm{c}} \mathrm{N}-\mathrm{C}_{\text {Phage }}$, and Phage $\mathrm{N}-\mathrm{C}_{\mathrm{Vc}}$ proteins shifted $\mathrm{P} v q m R$ DNA, whereas only the $\mathrm{VqmA}_{\text {Phage }}$ and ${ }_{\mathrm{Vc}} \mathrm{N}-\mathrm{C}_{\text {Phage }}$ proteins shifted Pqtip DNA (Fig 1D). Assessing the ratios of bound to total DNA across varying protein concentrations allowed us to calculate the relative binding affinities $\left(\mathrm{EC}_{50}\right)$ of the VqmA proteins for PvqmR and Pqtip DNA (S2A Fig). Our EMSA analyses show that 


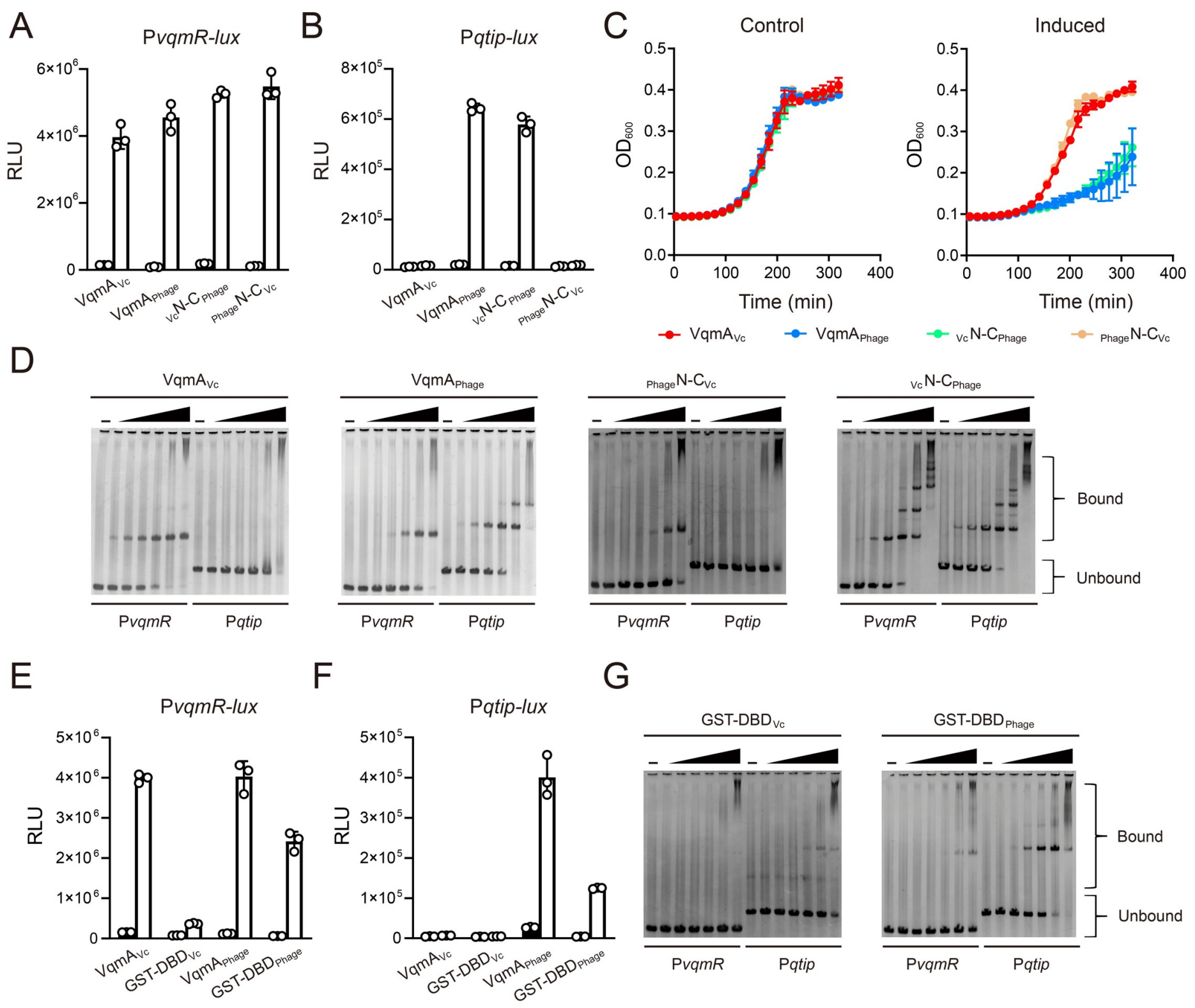

Fig 1. Promoter DNA-binding selectivity is conferred by the VqmA DBD. (A and B) Normalized reporter activity from $\Delta t d h$ E. coli harboring (A) PvqmR-lux or (B) Pqtip-lux and arabinose-inducible $\mathrm{VqmA}_{\mathrm{Vc}}, \mathrm{VqmA}_{\text {Phage, }} \mathrm{V}_{\mathrm{c}} \mathrm{N}-\mathrm{C}_{\text {Phage, }}$, or $\mathrm{Phage}_{\mathrm{N}} \mathrm{C}_{\mathrm{Vc}}$. Black, no arabinose; white, $0.2 \%$ arabinose. Data are represented as mean $\pm \mathrm{SD}$ (error bars) with $n=3$ biological replicates. (C) Growth curves of the $\Delta t d h \Delta v q m A_{V c} V$. cholerae harboring phage VP882 vqmA $A_{\text {Phage }}: \operatorname{Tn} 5$ and arabinose-inducible $\mathrm{VqmA}_{\mathrm{V}_{\mathrm{c}}}, \mathrm{VqmA}_{\text {Phage, }} \mathrm{V}_{\mathrm{c}} \mathrm{N}-\mathrm{C}_{\text {Phage }}$, or ${ }_{\text {Phage }} \mathrm{N}-\mathrm{C}_{\mathrm{Vc}}$ in medium lacking (Control) or containing $0.2 \%$ arabinose (Induced). (D) EMSAs showing binding of $\mathrm{V}_{\mathrm{m}} \mathrm{A}$ proteins to $\mathrm{P} v q m R$ and $\mathrm{P} q$ tip DNA. From left to right are, $\mathrm{VqmA}_{\mathrm{Vc}}, \mathrm{VqmA}_{\text {Phage, Phage }} \mathrm{N}-\mathrm{C}_{\mathrm{Vc}}$, and ${ }_{\mathrm{Vc}} \mathrm{N}-\mathrm{C}_{\mathrm{Phage}} .25 \mathrm{nM} \mathrm{P} v q m R$ or Pqtip DNA was used in all EMSAs with no protein (designated -) or 2-fold serial dilutions of proteins. The lowest and highest protein (dimer) concentrations are $18.75 \mathrm{nM}$ and $600 \mathrm{nM}$, respectively. (E and F) Normalized

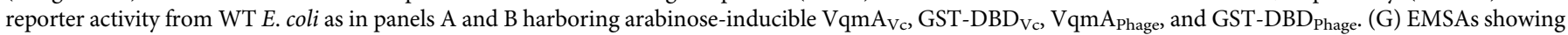
binding of GST-DBD $\mathrm{Vc}_{\mathrm{c}}$ and GST-DBD $\mathrm{Phage}_{\mathrm{f}}$ to $\mathrm{P} v q m R$ and Pqtip DNA. Probe and protein concentrations as in panel D.

https://doi.org/10.1371/journal.pgen.1009550.g001

Phage $\mathrm{N}-\mathrm{C}_{\mathrm{Vc}}$, like $\mathrm{VqmA}_{\mathrm{Vc}}$, only bound $\mathrm{P} v q m R$, but with an estimated $\sim 7$-fold lower affinity. Consistent with our previous findings, $\mathrm{VqmA}_{\mathrm{Phage}}$ bound $\mathrm{P} q$ tip about 3-fold more strongly than it bound $\mathrm{P} v q m R$ [5]. By contrast, ${ }_{\mathrm{V}} \mathrm{N}-\mathrm{C}_{\text {Phage }}$ showed a modest increase in its preference for Pqtip relative to that for $\mathrm{P} v q m R$, with binding to both promoters at a level similar to that with which VqmA $\mathrm{A}_{\text {Phage }}$ bound Pqtip. Indeed, in agreement with our $\mathrm{EC}_{50}$ measurements, when Pqtip and $\mathrm{P} v q m R$ DNA were supplied at equimolar concentrations in a competitive 
DNA-binding assay, lower amounts of $\mathrm{VqmA}_{\text {Phage }}$ and ${ }_{\mathrm{Vc}} \mathrm{N}-\mathrm{C}_{\text {Phage }}$ were required to shift $\mathrm{P} q$ tip DNA than to shift PvqmR DNA (S2B Fig). In conclusion and in agreement with our in vivo results, the respective DBD of each purified VqmA protein drives promoter selectively.

We next assayed the $\mathrm{VqmA}_{\mathrm{Vc}}$ and $\mathrm{Vqm}_{\text {Phage }} \mathrm{DBD}$ lacking their PAS domains $\left(\mathrm{DBD}_{\mathrm{Vc}}\right.$ and $\mathrm{DBD}_{\text {Phage }}$, respectively) for activation of PvqmR-lux and Pqtip-lux. Deletion of the PAS domains resulted in inactive proteins as neither DBD activated transcription ( $\mathrm{S} 3 \mathrm{~A}$ and $\mathrm{S} 3 \mathrm{~B}$ Fig, respectively), and likewise, EMSA analyses showed that neither DBD bound either promoter (S3C Fig). Gel filtration analyses indicated that the DBD proteins purified as monomers (S3D Fig), suggesting that the DBDs were unable to dimerize in the absence of their partner PAS domains. This result is consistent with previous findings that, in addition to sensing $\mathrm{DPO}$, the $\mathrm{VqmA}_{\mathrm{Vc}}$ PAS domain is responsible for dimerization $[9,11]$.

Transcriptional activity driven by HTH-containing proteins typically depends on dimer formation. Soluble glutathione S-transferase (GST) spontaneously forms a homodimer [12], and so GST can be employed as a substitute for native dimerization domains of proteins [13]. Thus, to examine the VqmA requirement for dimerization, we fused GST to the N-terminus of

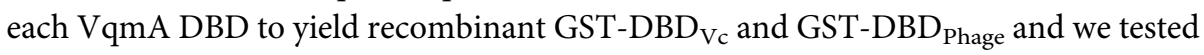
whether DNA-binding function was restored. Indeed, the GST-DBD proteins purified as dimers (S3D Fig). PvqmR-lux and Pqtip-lux expression analyses revealed that the DBDs, when fused to GST, regained function, with the caveat that the GST-DBD $\mathrm{Vc}_{\mathrm{V}}$ exhibited 10-fold reduced activity compared to wild-type (WT) $\mathrm{VqmA}_{\mathrm{Vc}}$ (Fig 1E). Importantly, the DNA-binding preferences mimicked those of the full-length proteins: GST-DBD ${ }_{\text {Phage }}$ activated both $\mathrm{P} v q m R$-lux and Pqtip-lux, whereas GST-DBD $\mathrm{Vc}_{\mathrm{c}}$ only activated PvqmR-lux (Fig $1 \mathrm{E}$ and $1 \mathrm{~F}$ ). Companion EMSA analyses showed that GST-DBD Phage bound Pqtip $\sim 5$-fold more strongly than it bound $\mathrm{P} v q m R$, whereas GST-DBD $\mathrm{Vc}_{\mathrm{c}}$ showed almost no binding to $\mathrm{P} v q m R$ and, unexpectedly, some weak binding could be detected to the Pqtip DNA (Fig 1G). We confirmed that purified GST alone did not bind either P $v q m R$ or Pqtip (S3E Fig). Given that the GST-DBD $\mathrm{Vc}_{\mathrm{c}}$ driven activation of Pqtip-lux was undetectable in vivo (Fig $1 \mathrm{~F}$ ), we presume that the observed in vitro GST-DBD $\mathrm{Vc}_{\mathrm{Vc}}$ binding to $\mathrm{P} q$ tip DNA is a consequence of the simplified context in which the EMSA is performed. Likely, the DNA:VqmA ratio in the EMSA is far higher than in cells, which, in the case of GST-DBD ${ }_{\mathrm{Vc}}$, fosters modest non-specific DNA binding. Taken together, our results show that VqmA promoter-binding selectivity is conferred by the DBD, and that dimerization is necessary.

\section{VqmA DNA-binding preferences can be inverted by exchanging key DNA sequences in PvqmR and Pqtip}

To study the VqmA promoter-binding asymmetry from the aspect of the DNA, our next goal was to identify the critical DNA sequence within Pqtip that prevents $\mathrm{VqmA}_{\mathrm{Vc}}$ from binding. In the phage VP882 genome, Pqtip resides between $v q m A_{\text {Phage }}$ and qtip and $V q m A_{\text {Phage }}$ activates its own and qtip expression, suggesting that $\mathrm{VqmA}_{\text {Phage }}$ binding may involve both DNA strands. Similarly, $\mathrm{VqmA}_{\mathrm{Vc}}$ has been shown to interact with both strands of $\mathrm{P} v q m R$ [11]. Thus, in each case, both DNA strands need to be considered (Fig 2A). Previous work revealed that the critical region in $\mathrm{P} v q m R$ required for $\mathrm{VqmA}_{\mathrm{Vc}}$ binding is - AGGGGGGATTTCCCCCCT$[2,11]$. The corresponding fragment from Pqtip, but on the opposite DNA strand, -TAGGGG GAAAAATACCCT-, possesses $\sim 56 \%$ sequence identity to this region suggesting it could be the key stretch of DNA that drives $\mathrm{VqmA}_{\text {Phage }}$ promoter selection. The highest divergence in the two promoters is in the central 6 nucleotides: "-AAAATA-" in Pqtip and "-TTTCCC-" in $\mathrm{P} v q m R$. We synthesized DNA probes in which we exchanged the "-AAAATA-" in Pqtip with "-TTTCCC-" from PvqmR and tested $\mathrm{VqmA}_{\mathrm{Vc}}$ and $\mathrm{VqmA}_{\text {Phage }}$ binding by EMSA analysis. 
A

PvqmR
Pqtip (Re

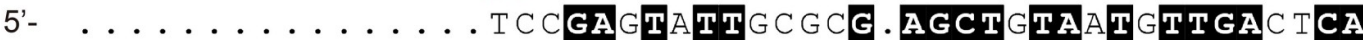

5'- GAATACACTCCTTGTAAGTGATTGTTATAAGGAGCTTTATTCTTGATGCA $-1$

$\mathrm{GG}-\mathrm{N}_{6}-\mathrm{CC}$

PvqmR

Pqtip (Rev)

5'- A $\mathbf{A} C \mathbf{A} \mathbf{A} T \mathrm{~A}$

5'- TATAAGA

G_GGGGA T T T C C CCC T T T T T T T GTAC C C G

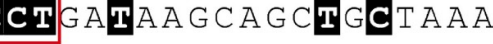
$-3^{\prime}$ $\mathrm{GG}-\mathrm{N}_{9}-\mathrm{CC}$ Pqtip (Rev)
5'-
A C

B
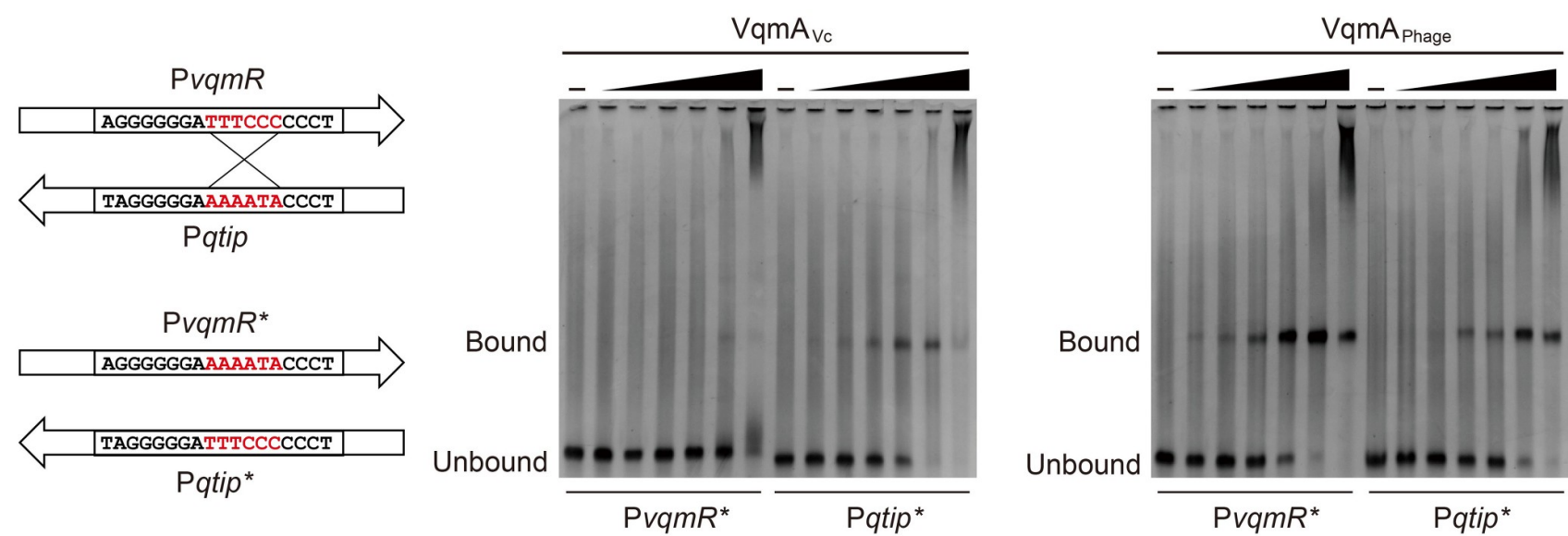

C
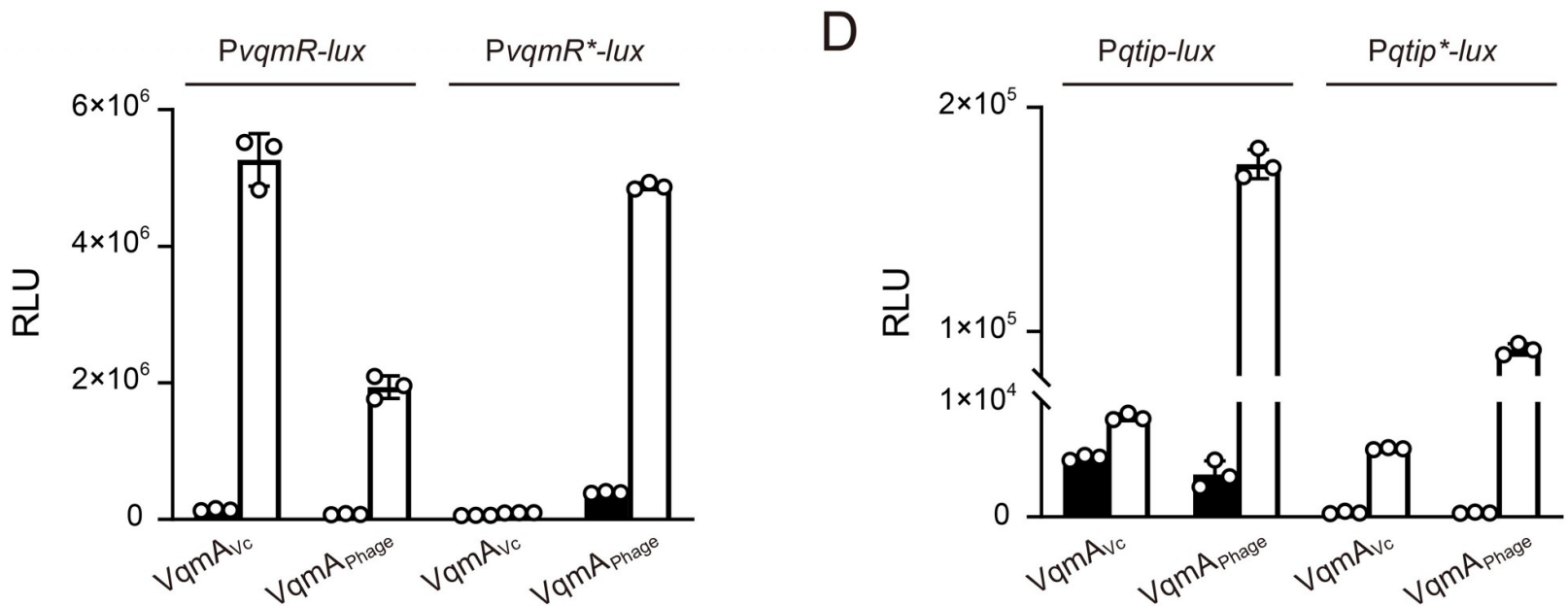

Fig 2. Promoter selectivity is reversed by exchanging key nucleotide fragments. (A) DNA sequence alignment (ClustalW) of PvqmR and Pqtip. The reverse strand of Pqtip is shown. Numbering indicates positions relative to the transcription start sites. Identical nucleotides are designated with black shading. The reported 18-bp DNA stretch in $\mathrm{P} v q m R$ required for $\mathrm{VqmA}_{\mathrm{Vc}}$ to bind $(2,11)$ and the corresponding region in Pqtip are highlighted in the red box. The GG- $\mathrm{N}_{6}$ CC palindrome in PvqmR $(2,11)$ and the recently identified GG- $\mathrm{N}_{9}$-CC palindrome in Pqtip (15) are indicated above and below the red box, respectively. (B) EMSAs showing binding of the designated VqmA proteins to P $v q m R^{*}$ and Pqtip ${ }^{*}$ DNA. The cartoon at the left illustrates the key sequences exchanged in the probes. Probe and protein concentrations as in Fig 1D. (C) Normalized reporter activity from $\Delta t d h$ E. coli harboring P $v q m R$-lux or P $v q m R^{*}-l u x$ and arabinoseinducible $\mathrm{VqmA}_{\mathrm{Vc}}$ or $\mathrm{VqmA}_{\text {Phage }}$. Black, no arabinose; white, $0.2 \%$ arabinose. Data are represented as mean $\pm \mathrm{SD}$ (error bars) with $n=3$ biological replicates. (D) As in C for Pqtip-lux or Pqtip*-lux. 
We call these probes $\mathrm{P} v q m R^{*}$ and $\mathrm{P} q t p^{*}$, respectively. Indeed, promoter DNA-binding specificity was exchanged: $\mathrm{VqmA}_{\mathrm{V}_{c}}$ shifted Pqtip*, whereas it only weakly shifted $\mathrm{P} v q m R^{*}$ (Fig $2 \mathrm{~B}$ ). $\mathrm{VqmA}_{\text {Phage }}$ bound to $\mathrm{P} v q m R^{*}$ twice as strongly as it bound to Pqtip*, showing the opposite preference for the two synthetic promoters compared to the native promoters (Fig 2B). $\mathrm{P} v q m R^{*}$-lux and Pqtip*-lux transcriptional fusions mimicked the EMSA results: $\mathrm{VqmA}_{\mathrm{Vc}}$ only activated expression of $\mathrm{P} q t i p^{*}$-lux, whereas $\mathrm{VqmA}_{\text {Phage }}$ activated expression of $\mathrm{P} v q m R^{*}$-lux and Pqtip*-lux (Fig 2C and 2D). Thus, this 6-nucleotide stretch is the key sequence that determines the DNA-binding specificity for the two VqmA proteins. Moreover, the presence of the -AAAATA- nucleotide sequence in Pqtip is sufficient to prevent $\mathrm{VqmA}_{\mathrm{Vc}}$ from activating transcription of Pqtip.

\section{Protein sequence-guided mutagenesis reveals that residue E194 in phage VP882 Vqm $A_{P h a g e}$ and the equivalent $\mathrm{A} 192$ residue in $V$. cholerae $\mathrm{VqmA}_{\mathrm{Vc}}$ contribute to specificity for Pqtip}

We considered two possible mechanisms that could underpin the asymmetric VqmA DNAbinding patterns: phage VP882 VqmA $A_{\text {Phage }}$ could possess a feature that relaxes its DNA-binding specificity, and/or $V$. cholerae $\mathrm{VqmA}_{\mathrm{Vc}}$ could possess a feature that restricts its DNA-binding ability. To distinguish between these possibilities, we first probed which residues drive $V_{q m A} A_{\text {Phage }}$ interactions with Pqtip but do not contribute to interactions with $\mathrm{P} v q m R$. To do this, we performed site-directed mutagenesis of $\mathrm{VqmA}_{\text {Phage }}$ with the goal of identifying mutants that fail to bind Pqtip but retain binding to P $v q m R$. Charged residues in HTH motifs typically mediate interactions between VqmA-type transcription factors and DNA, and indeed, both VqmA HTHs are enriched in positively-charged amino acids $[9,11,14]$. Sequence alignment of the HTHs in $\mathrm{VqmA}_{\mathrm{Phage}}$ and $\mathrm{VqmA}_{\mathrm{Vc}}$ revealed four obvious differences in charged residues that could underlie the DNA-binding asymmetry between the two proteins (S1 Fig). We mutated those residues in $\mathrm{VqmA}_{\mathrm{Phage}}$ to the corresponding $\mathrm{VqmA}_{\mathrm{Vc}}$ residues. The changes are: $\mathrm{VqmA}_{\text {Phage }}{ }^{\mathrm{K} 176 \mathrm{Q}}, \mathrm{VqmA}_{\text {Phage }}{ }^{\mathrm{R} 184 \mathrm{I}}, \mathrm{VqmA}_{\text {Phage }}{ }^{\mathrm{I} 93 \mathrm{E}}$, and $\mathrm{VqmA}_{\text {Phage }}{ }^{\mathrm{E} 194 \mathrm{~A}}$. To test the combined effect of these mutations on VqmA $\mathrm{A}_{\text {Phage }}$ DNA-binding function, we also

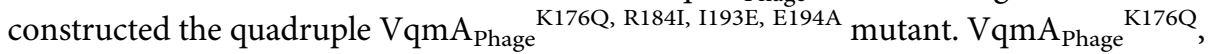
$\mathrm{VqmA}_{\text {Phage }}{ }^{\mathrm{R} 184 \mathrm{I}}$, VqmA $\mathrm{Phage}^{\mathrm{II}}{ }^{\mathrm{E}}$ retained the ability to induce phage lysis showing that in vivo binding to Pqtip was not eliminated (Fig 3A). VqmA ${ }_{\text {Phage }}{ }^{\mathrm{E} 194 \mathrm{~A}}$ induced only low-level cell lysis suggesting that, while binding to Pqtip is not eliminated, it is compromised (Fig 3A). Analysis of $\mathrm{P} v q m R$-lux and $\mathrm{P} q$ tip-lux expression revealed that all four $\mathrm{VqmA}_{\mathrm{Phage}}$ single point mutants possessed levels of activity within 2-fold of that of WT PvqmR-lux. By contrast, they displayed $\sim 2-7$-fold reductions in Pqtip-lux activity, with $\mathrm{VqmA}_{\text {Phage }}{ }^{\mathrm{E} 194 \mathrm{~A}}$ being the least active (Fig 3B and $3 \mathrm{C}$, respectively). The quadruple mutant was unable to induce phage lysis in a $V$. cholerae lysogen and it did not activate PvqmR-lux or Pqtip-lux expression showing it is defective in binding to both promoters (Fig 3A-3C). Western blot analysis demonstrated that all of the $\mathrm{VqmA}_{\text {Phage }}$ variants were produced at levels similar to WT in both $V$. cholerae and E. coli (S4A Fig). Thus, our results indicate that, among these charged residues, only the $\mathrm{VqmA}_{\text {Phage }}$ residue E194 in the HTH motif plays a role in $\mathrm{VqmA}_{\text {Phage }}$ selection of Pqtip.

While the residues we mutated in the phage VP882 VqmA $A_{\text {Phage }}$ HTH motif do not dramatically perturb site-specific recognition of Pqtip, the corresponding residues in the V. cholerae $\mathrm{VqmA}_{\mathrm{Vc}} \mathrm{HTH}$ motif could nonetheless restrict its capacity to bind Pqtip. Therefore, we also mutated the analogous $\mathrm{VqmA}_{\mathrm{Vc}}$ residues to the corresponding $\mathrm{VqmA}_{\mathrm{Phage}}$ residues. We made:

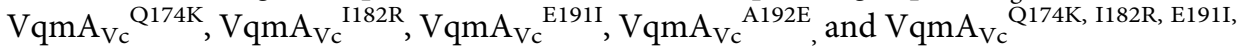
${ }^{\mathrm{A} 192 \mathrm{E}}$. Here, our goal was to test whether the variants gained the ability to bind Pqtip. Only $\mathrm{VqmA}_{\mathrm{Vc}}{ }^{\mathrm{A} 192 \mathrm{E}}$ induced a modest level of lysis in the V. cholerae lysogen, whereas all other 
A

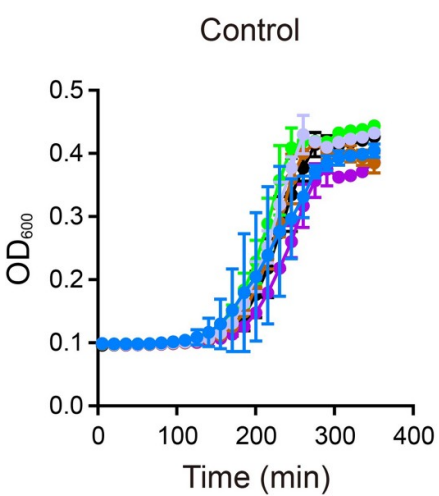

B

Induced

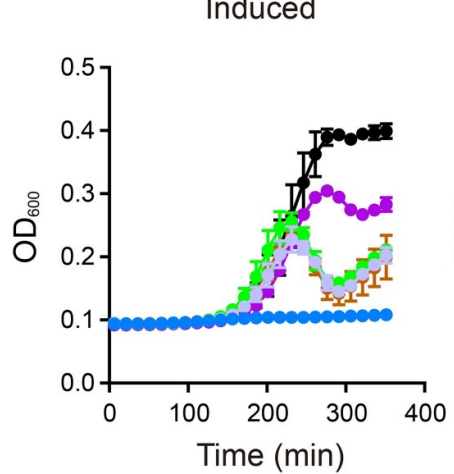

PvqmR-lux

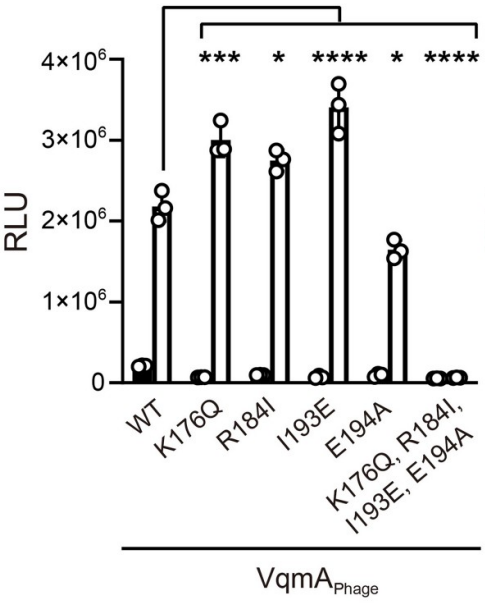

E
C Pqtip-lux

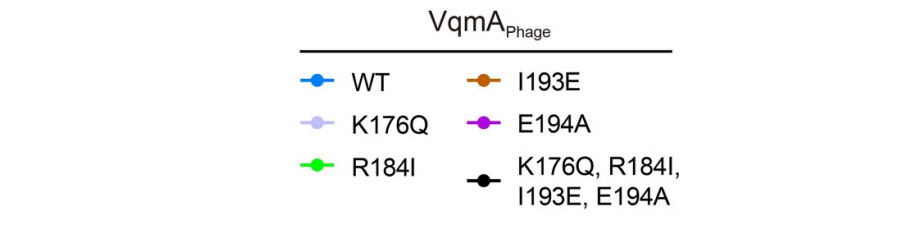

D

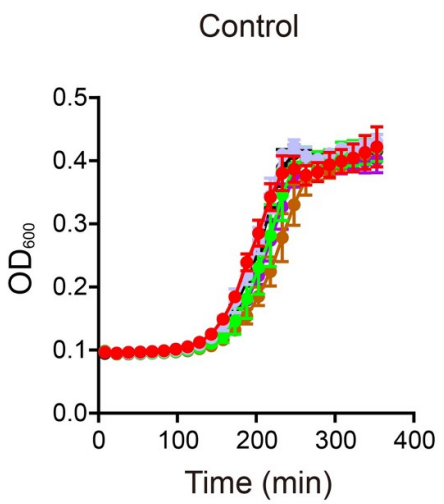

Induced

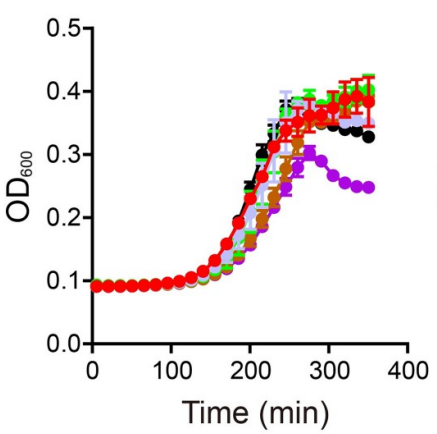

$\operatorname{VqmA}_{\mathrm{Vc}}$

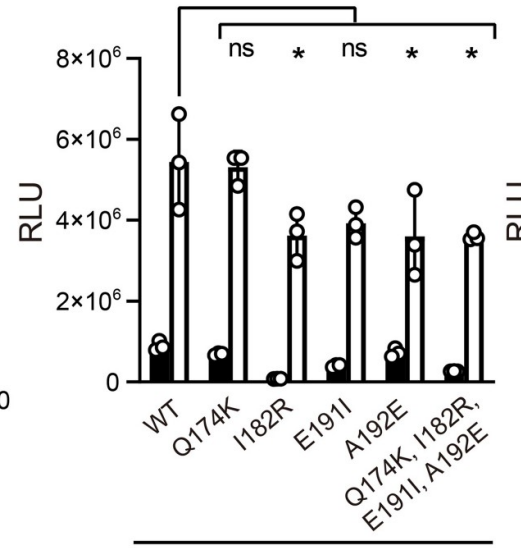

$\mathrm{VqmA}_{\mathrm{vo}}$

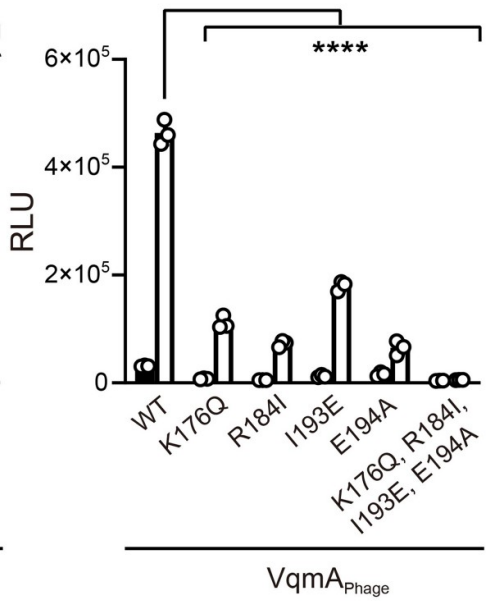

F
PvqmR-Iux

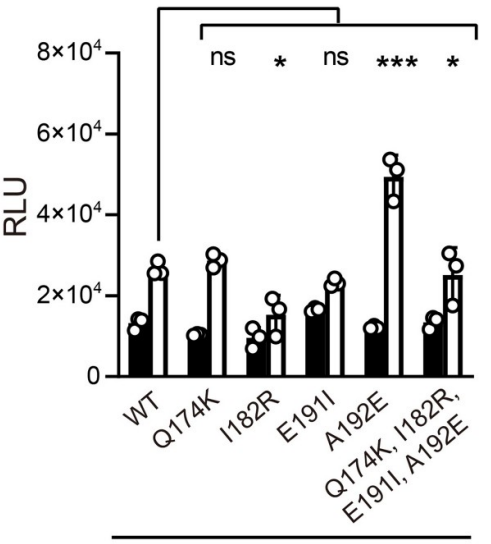

$\mathrm{VqmA}_{\mathrm{vc}}$

$\rightarrow$ Q174K $\rightarrow$ A192E

Q174K, I182R

E191I, A192E

Fig 3. Vqm $A_{\text {Phage }}$ residue E194 and the corresponding $\mathrm{VqmA}_{\mathrm{Vc}}$ residue A192 contribute to specificity for binding to Pqtip. (A) Growth curves of $\Delta t d h$ $\Delta v q m A_{V c}$ V. cholerae harboring phage VP882 vqmA $A_{\text {Phage }}:$ Tn5 and the indicated 3xFLAG-VqmA Phage alleles in medium lacking (Control) or containing $0.2 \%$ arabinose (Induced). (B and C) Normalized reporter activity from $\Delta t d h$ E. coli harboring (B) PvqmR-lux or (C) Pqtip-lux and the indicated arabinose-inducible $3 x$ FLAG-VqmA $A_{\text {Phage }}$ alleles. Black, no arabinose; white, $0.2 \%$ arabinose. Data are represented as mean \pm SD (error bars) with $n=3$ biological replicates. (D) Growth curves of $\Delta t d h \Delta v q m A_{V c} V$. cholerae harboring phage VP882 vqmA $A_{\text {Phage: }}: T n 5$ and the indicated 3xFLAG-VqmA $A_{V c}$ alleles in medium lacking (Control) or containing $0.2 \%$ arabinose (Induced). (E and F) Normalized reporter activity from $\Delta t d h$ E. coli harboring (E) PvqmR-lux or (F) Pqtip-lux and the indicated arabinose-inducible $3 \mathrm{xFLAG}-\mathrm{VqmA}_{\mathrm{Vc}}$ alleles. Black, no arabinose; white, $0.2 \%$ arabinose. Data are represented as mean $\pm \mathrm{SD}$ (error bars) with $n=3$ biological replicates. ns $=$ not significant, ${ }^{* * *} P<0.0001,{ }^{* * *} P<0.0005,{ }^{* *} P<0.005,{ }^{*} P<0.05$ in one-way ANOVA compared to WT VqmA proteins.

https://doi.org/10.1371/journal.pgen.1009550.g003

$\mathrm{VqmA}_{\mathrm{Vc}}$ variants failed to do so (Fig 3D). All of the $\mathrm{VqmA}_{\mathrm{Vc}}$ variants drove the WT level of P vqmR-lux activity (Fig 3E). VqmA ${ }_{\mathrm{Vc}}{ }^{\mathrm{A} 192 \mathrm{E}}$ generated low but detectable Pqtip-lux expression, while the other $\mathrm{VqmA}_{\mathrm{Vc}}$ variants $\operatorname{did}$ not (Fig $3 \mathrm{~F}$ ). The $\mathrm{VqmA}_{\mathrm{Vc}}$ variants were produced at similar levels to WT VqmA $\mathrm{V}_{\mathrm{Vc}}$ in $V$. cholerae and E. coli (S4B Fig). We conclude that, among the tested residues, only $\mathrm{A} 192$ plays a role in preventing $\mathrm{VqmA}_{\mathrm{Vc}}$ from binding Pqtip. 
Our mutagenesis analyses for $\mathrm{VqmA}_{\mathrm{Vc}}$ are consistent with our analyses for $\mathrm{VqmA}_{\text {Phage: }}$ The residue at the $192^{\text {nd }}$ position in $V$. cholerae $\mathrm{VqmA}_{\mathrm{Vc}}$ and the analogous residue at the $194^{\text {th }}$

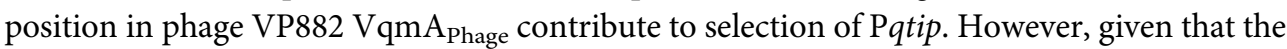
A192E substitution in $\mathrm{VqmA}_{\mathrm{Vc}}$ results in only partial activation of Pqtip expression, and the

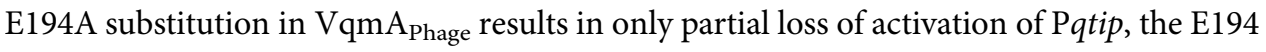
residue in $\mathrm{VqmA}_{\text {Phage }}$ cannot be the sole amino acid responsible for the preference $\mathrm{VqmA}_{\text {Phage }}$ shows for Pqtip. Rather, additional residues in $\mathrm{VqmA}_{\text {Phage }}$ must participate in conferring specificity.

\section{Random mutagenesis of the VqmA $A_{\text {Phage }}$ DBD reveals that residues G201, A202, E207, and M211 are required for VqmA $A_{\text {Phage }}$ to bind Pqtip but are dispensable for binding PvqmR}

Our protein sequence-guided approach did not reveal the primary mechanism underlying promoter-binding specificity for either of the VqmA proteins. We therefore performed a

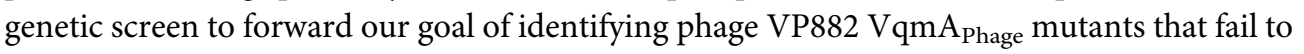
bind Pqtip but retain the ability to bind $\mathrm{P} v q m R$. We constructed a library of random mutations in the region of $v q m A_{\text {Phage }}$ encoding the DBD in the context of the full-length gene, cloned them into a plasmid under an arabinose-inducible promoter, and introduced them into $\Delta t d h$ $\Delta v q m A_{V c} V$. cholerae harboring $\mathrm{P} v q m R$-lux on the chromosome and lysogenized by phage

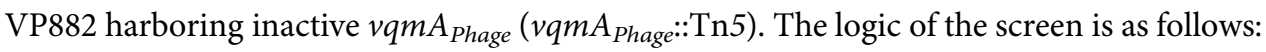
When propagated on agar plates supplemented with arabinose, $V$. cholerae exconjugants harboring $v q m A_{\text {Phage }}$ alleles possessing reasonable Pqtip-binding activity will lyse because those $\mathrm{VqmA}_{\text {Phage }}$ proteins will bind Pqtip on the phage VP882 genome and launch the phage lytic cascade (S5 Fig). Such exconjugants will die and thus be eliminated from the screen. Exconjugants that survive but carry $v q m A_{\text {Phage }}$ null alleles will produce no light because those $\mathrm{VqmA}_{\text {Phage }}$ proteins will fail to bind $\mathrm{P} v q m R$-lux, so they also can be eliminated from the screen. The $v q m A_{\text {Phage }}$ alleles of interest to us are those that are maintained in surviving exconjugants (because they encode proteins that cannot bind Pqtip) and produce light (because they encode proteins that can bind PvqmR-lux).

Our screen yielded the following mutants: VqmA $A_{\text {Phage }}{ }^{G 201 D}, V_{\text {qmA }} A_{\text {Phage }}$ G201R, $\mathrm{VqmA}_{\text {Phage }}{ }^{\mathrm{A} 202 \mathrm{~V}}, \mathrm{VqmA}_{\text {Phage }}{ }^{\mathrm{E} 207 \mathrm{~K}}, \mathrm{VqmA}_{\text {Phage }}{ }^{\text {E207V }}$, and $\mathrm{VqmA}_{\text {Phage }}{ }^{\mathrm{M} 211 \mathrm{~K}}$ (Fig $\left.4 \mathrm{~A}\right)$. To verify that these VqmA $A_{\text {Phage }}$ mutants were indeed defective in binding Pqtip, we individually transformed them into $\Delta t d h$ E. coli carrying the Pqtip-lux reporter or the PvqmR-lux reporter and measured light production. All variants retained WT capability to activate PvqmR-lux, but they did not harbor WT capability to activate Pqtip-lux expression $(>10$-fold reductions in activity) (Fig 4B and 4C, respectively). Thus, any residual Pqtip binding by these mutant $\mathrm{VqmA}_{\text {Phage }}$ proteins is insufficient to induce host-cell lysis in the phage VP882 lysogen (Fig $4 \mathrm{~A}$ ). We verified that the $\mathrm{VqmA}_{\text {Phage }}$ variants are produced at the same level as $\mathrm{WT} V \mathrm{Vqm} \mathrm{A}_{\text {Phage }}$ in $V$. cholerae and E. coli (S4C Fig). According to the protein sequence alignment, $\mathrm{VqmA}_{\text {Phage }}$ residues (175-200) corresponding to positions 173-198 in $\mathrm{VqmA}_{\mathrm{Vc}}$ comprise the $\mathrm{VqmA}_{\text {Phage }}$ HTH motif (S1 Fig). Thus, the residues identified in the mutagenesis (G201, A202, E207, and M211) are located C-terminal to the $\mathrm{VqmA}_{\text {Phage }} \mathrm{HTH}$ motif. Mapping the analogous $V$. cholerae $\mathrm{VqmA}_{\mathrm{Vc}}$ residues (G199, A200, Q205, and L209) to the DPO-VqmA $\mathrm{V}_{\mathrm{Vc}} \mathrm{P} v q m R$ structure (there is no DPO-VqmA $\mathrm{Phage}_{\mathrm{P}} \mathrm{P}$ tip structure) also shows that all of these residues cluster in a flexible loop region and helix adjacent to, but distinct from the HTH motif that directly contacts DNA (Figs 4D and S1). Surprisingly, the residues identified in the VqmA $A_{\text {Phage }}$ mutagenesis are either identical ( $\mathrm{VqmA}_{\text {Phage }} \mathrm{G} 201$ and $\mathrm{A} 202$ versus $\mathrm{VqmA}_{\mathrm{Vc}} \mathrm{G} 199$ and $\left.\mathrm{A} 200\right)$ or similar $\left(V_{\text {qmA }} A_{\text {Phage }}\right.$ E207 and M211 versus VqmA $A_{V c}$ Q205 and L209) between VqmA $A_{\text {Phage }}$ 
A

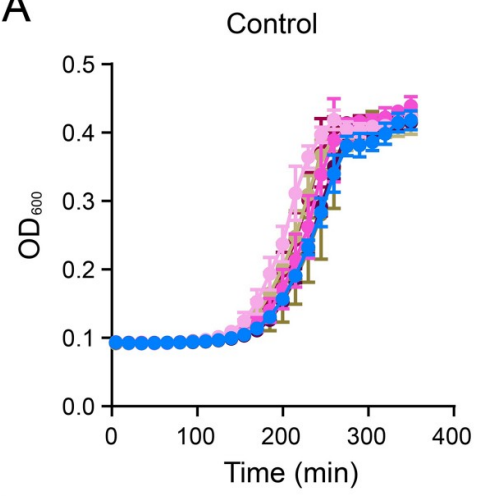

B

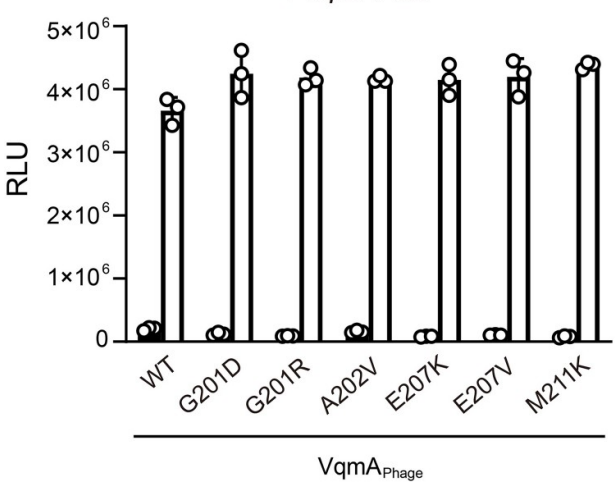

PvqmR-lux

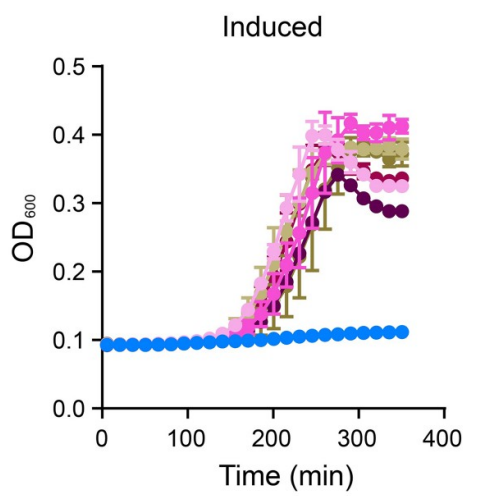

C

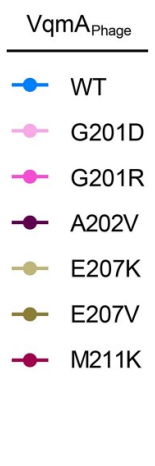

Pqtip-lux

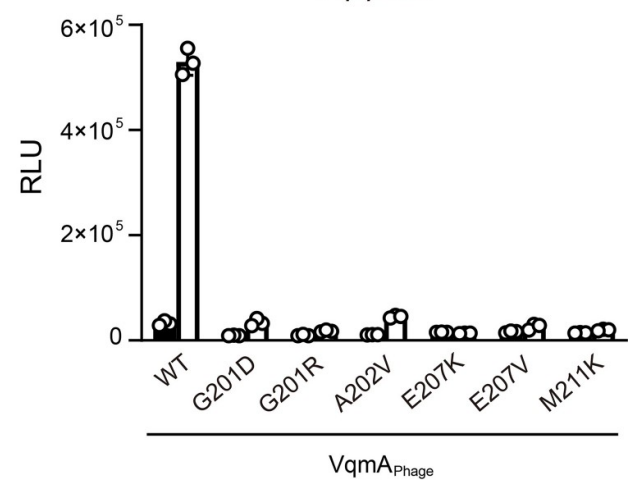

D
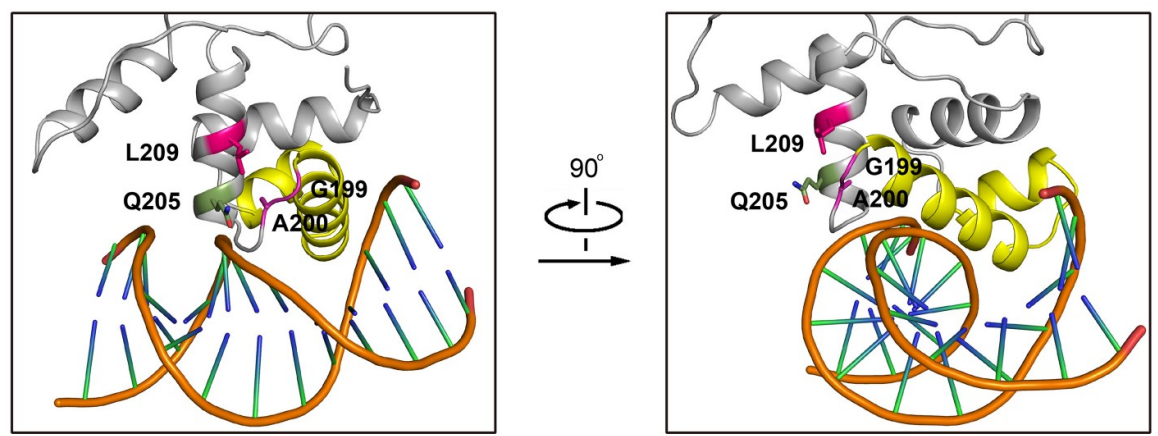

Fig 4. VqmA $A_{\text {Phage }}$ residues G201, A202, E207, and M211 are required for binding to Pqtip. (A) Growth curves of $\Delta t d h \Delta v q m A_{V c}$ V. cholerae harboring phage VP882 vqmA $A_{\text {Phage }}: T n 5$ and the indicated 3xFLAG-VqmA $A_{\text {Phage }}$ alleles in medium lacking (Control) or containing $0.2 \%$ arabinose (Induced). (B and C) Normalized reporter activity from $\Delta t d h$ E. coli harboring (B) PvqmR-lux or (C) Pqtip-lux and the indicated arabinose-inducible 3xFLAG-VqmA Phage $_{\text {alleles. }}$ Black, no arabinose; white $0.2 \%$ arabinose. Data are represented as mean \pm SD (error bars) with $n=3$ biological replicates. (D) Close up views of the $\mathrm{DBD}$ from the crystal structure of $\mathrm{DPO}-\mathrm{VqmA}_{\mathrm{Vc}}$ bound to $\mathrm{P} v q m R$ (PDB: 6ide, protein in gray with the HTH motif in yellow, and the DNA in orange). The color scheme for $\mathrm{VqmA}_{\mathrm{Vc}}$ residues G199, A200, Q205, and L209 mirrors that used in panel A.

https://doi.org/10.1371/journal.pgen.1009550.g004

and $\mathrm{VqmA}_{\mathrm{Vc}}$. To test whether possession of the similar residues is sufficient to confer DNAbinding specificity for Pqtip, we constructed $\mathrm{VqmA}_{\mathrm{Vc}_{\mathrm{c}}}{ }^{\mathrm{Q} 205 \mathrm{E}}$ and $\mathrm{VqmA}_{\mathrm{Vc}}{ }^{\mathrm{L} 209 \mathrm{M}}$ and tested their DNA-binding functions as above. $\mathrm{VqmA}_{\mathrm{Vc}^{\mathrm{Q}}}{ }^{\mathrm{Q} 205 \mathrm{E}}$ and $\mathrm{VqmA}_{\mathrm{Vc}}{ }^{\mathrm{L} 209 \mathrm{M}}$, like WT VqmA $\mathrm{V}_{\mathrm{Vc}}$, activated $\mathrm{P} v q m R$-lux but failed to activate Pqtip-lux (S6A and S6B Fig, respectively). We make the following four conclusions from these findings: 1) There are at least four residues (G201, A202, E207, and M211) required for VqmA Phage to recognize Pqtip DNA. 2) Because the 
VqmA $_{\text {Phage }}$ G201D, G201R, A202V, E207K, E207V, and M211K variants exhibit WT binding to $\mathrm{P} v q m R$, the substitutions at these four residues must not significantly affect $\mathrm{P} v q m R$ recognition. 3) Because these residues are conserved or similar between $\mathrm{VqmA}_{\text {Phage }}$ and $\mathrm{VqmA}_{\mathrm{Vc}}$, one would expect $\mathrm{VqmA}_{\mathrm{Vc}}$ to have the capacity to bind Pqtip. 4) However, because $\mathrm{VqmA}_{\mathrm{Vc}}$ in fact does not bind Pqtip, $\mathrm{VqmA}_{\mathrm{Vc}}$ likely possesses an additional feature that resides elsewhere in the protein that prevents $\mathrm{P} q$ tip binding from occurring.

\section{The restrictive element that prevents $\mathrm{VqmA}_{\mathrm{Vc}}$ from binding Pqtip is located in its HTH motif and the adjacent C-terminal region of 25 residues}

To test the hypothesis that a feature in the $\mathrm{VqmA}_{\mathrm{Vc}} \mathrm{DBD}$ restricts its DNA-binding capacity to $\mathrm{P} v q m R$, we performed a genetic screen aimed at identifying $\mathrm{VqmA}_{\mathrm{Vc}}$ mutants capable of activating Pqtip-lux expression. To do this, we constructed a library of random $v q m A_{V c} \mathrm{DBD}$ alleles containing, on average, 1-2 substitutions, and we cloned them into a plasmid under an arabinose-inducible promoter. The library was transformed into the $\Delta t d h$ E. coli strain harboring the Pqtip-lux reporter and transformants were propagated on plates containing arabinose. We screened $\sim 10,000$ transformants for colonies that produced light indicating that they contained $\mathrm{VqmA}_{\mathrm{Vc}}$ proteins that activated Pqtip-lux. This strategy yielded no such transformants. Several possibilities could explain our result: We did not screen sufficient numbers of mutants, the mutagenesis did not yield the crucial change, or no alteration of a single residue can enable $\mathrm{VqmA}_{\mathrm{Vc}}$ binding to Pqtip.

We expanded our search for the DNA-binding restrictive element present in $\mathrm{VqmA}_{\mathrm{Vc}}$ by assessing whether a particular region in the $\mathrm{VqmA}_{\mathrm{Vc}} \mathrm{DBD}$ constrains promoter binding to $\mathrm{P} v q m R$. To do this, we constructed five $\mathrm{VqmA}_{\mathrm{Vc}}$ mosaic proteins by replacing $\sim 20-30$ residues in the $V$. cholerae $\mathrm{VqmA}_{\mathrm{Vc}} \mathrm{DBD}$ with the corresponding residues from the phage VP882

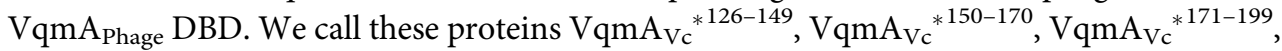

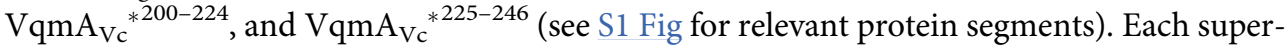
script denotes the $\mathrm{VqmA}_{\mathrm{Vc}}$ amino acid residues that have been replaced by the corresponding residues from $V q m A_{\text {Phage. }}$. In all the mosaics, either the intact $\mathrm{VqmA}_{\mathrm{Vc}} \mathrm{HTH}$ or the intact $\mathrm{VqmA}_{\text {Phage }} \mathrm{HTH}$ was present. For reference, the $\mathrm{VqmA}_{\mathrm{Vc}} \mathrm{HTH}$ motif consists of residues 173 to 198 and the VqmA $A_{\text {Phage }} \mathrm{HTH}$ spans residues 175 to 200 . We tested the mosaic $\mathrm{VqmA}_{\mathrm{Vc}}$ proteins for activation of the PvqmR-lux and Pqtip-lux reporters. The DNA specificity of all the $\mathrm{VqmA}_{\mathrm{Vc}}$ mosaics mimicked WT VqmA $\mathrm{V}_{\mathrm{Vc}}$ as $\mathrm{P} v q m R$-lux was expressed but Pqtip-lux was not (Fig 5A and 5B, respectively). We confirmed that the mosaic $\mathrm{VqmA}_{\mathrm{Vc}}$ proteins are expressed at levels similar to WT VqmA $A_{\mathrm{Vc}}$ (S7 Fig). Our results suggest that the feature that prevents $V$. cholerae $\mathrm{VqmA}_{\mathrm{Vc}}$ from binding to Pqtip is larger than the regions delineated by any of the $\mathrm{VqmA}_{\mathrm{Vc}}$ mosaics, or it could be that multiple patches in the $\mathrm{VqmA}_{\mathrm{Vc}} \mathrm{DBD}$ that are not contiguous in amino acid sequence are responsible.

Pinpointing non-contiguous regions that could, together, contain the $\mathrm{VqmA}_{\mathrm{Vc}}$ restrictive element is challenging. However, testing for a larger contiguous expanse that could contain the putative restrictive element is straightforward. Thus, we constructed two additional $V$. cholerae $\mathrm{VqmA}_{\mathrm{Vc}}$ mosaic proteins. In one construct, called $\mathrm{VqmA}_{\mathrm{Vc}^{* 150-199}}$, we introduced the $\mathrm{VqmA}_{\text {Phage }} \mathrm{HTH}$ along with the immediate $\mathrm{N}$-terminal 25 amino acids in place of the corresponding $\mathrm{VqmA}_{\mathrm{Vc}}$ region. Second, in a construct called $\mathrm{VqmA}_{\mathrm{Vc}_{\mathrm{c}}}{ }^{* 171-224}$, we introduced the $\mathrm{VqmA}_{\text {Phage }} \mathrm{HTH}$ together with the immediate C-terminal 25 amino acid stretch in place of that $\mathrm{VqmA}_{\mathrm{Vc}}$ region. $\mathrm{VqmA}_{\mathrm{Vc}^{* 150-199}}$ and $\mathrm{VqmA}_{\mathrm{Vc}^{* 171-224}}$ activated $\mathrm{P} v q m R$-lux to approximately WT levels, whereas only $\mathrm{VqmA}_{\mathrm{Vc}^{*}}{ }^{* 171-224}$ activated Pqtip-lux, albeit weakly (Fig 5C and $5 \mathrm{D}$, respectively). Consistent with this result, $\mathrm{VqmA}_{\mathrm{Vc}}{ }^{* 171-224}$ induced partial lysis in the $V$. cholerae phage VP882 lysogen (Fig 5E). $\mathrm{VqmA}_{\mathrm{Vc}^{* 171-224}}$ was produced at levels similar to 
A

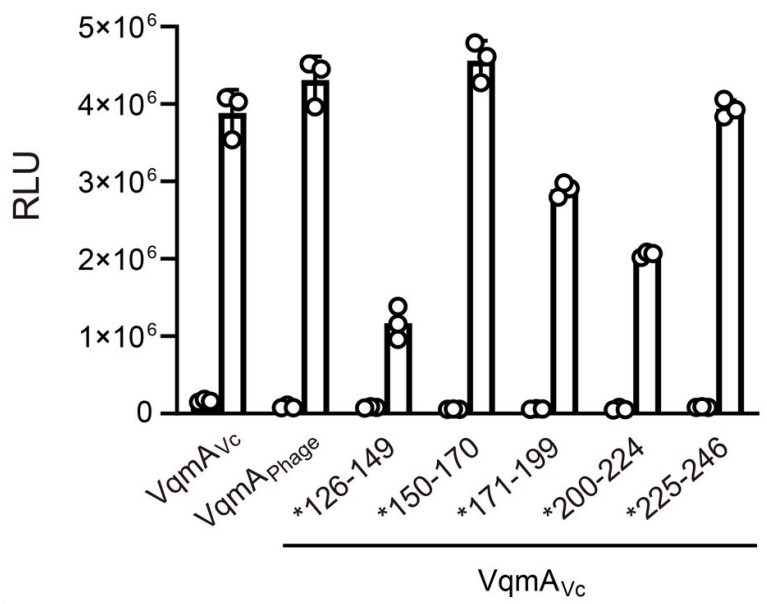

C

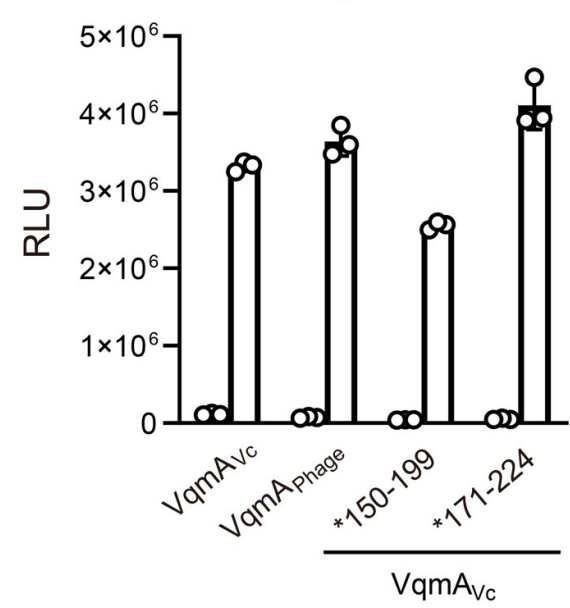

E

Control

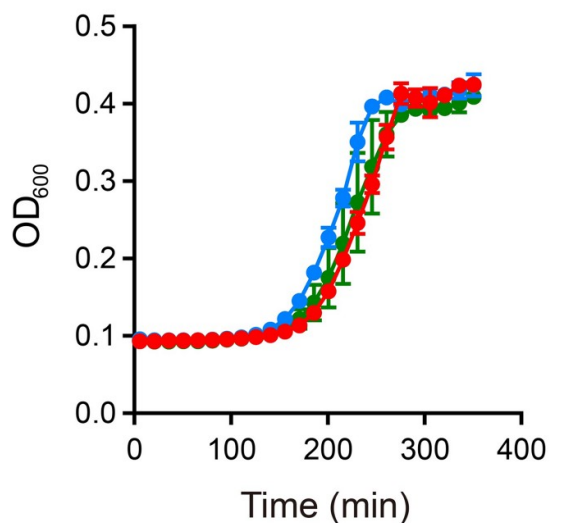

B Pqtip-lux

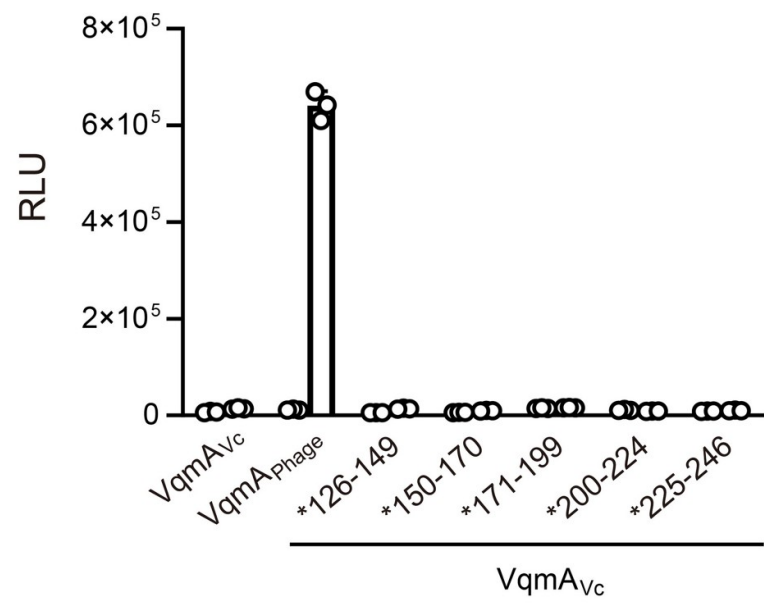

Pqtip-lux

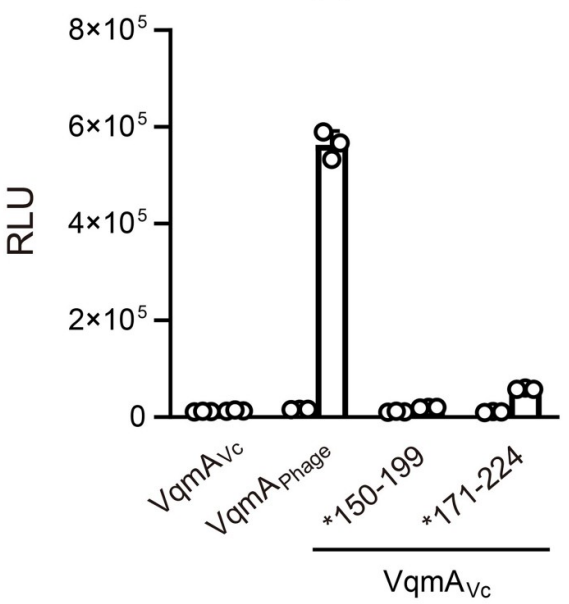

Induced

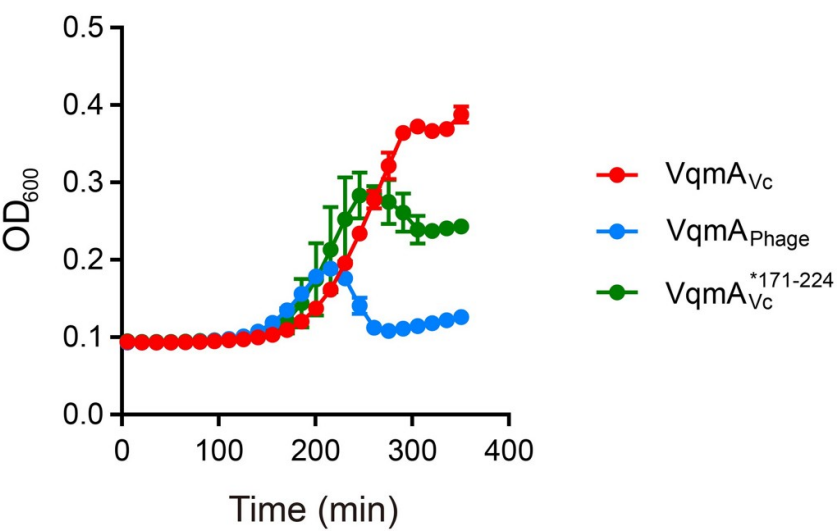

Fig 5. The Vqm $A_{V c}$ HTH motif and the immediate C-terminal 25 residues, together, constrain binding to PvqmR. (A-D) Normalized reporter activity from $\Delta t d h E$. coli harboring (A and C) P $v q m R$-lux or (B and D) Pqtip-lux and arabinose-inducible VqmA $\mathrm{V}_{\mathrm{V} c}$, VqmA $\mathrm{Phage}_{\mathrm{P}}$ or the indicated $\mathrm{VqmA}_{\mathrm{Vc}}$ allele. Data are represented as mean $\pm \mathrm{SD}$ (error bars) with $n=3$ biological replicates. Black, no arabinose; white, $0.2 \%$ arabinose. (E) Growth curves of $\Delta t d h \Delta v q m A_{V c} V$. cholerae harboring phage VP882 vqmA $A_{P h a g e}: \operatorname{Tn} 5$ and $\mathrm{VqmA}_{\mathrm{Vc}}, \mathrm{VqmA}_{\mathrm{Phage}}$, or $\mathrm{VqmA}_{\mathrm{Vc}} * 171-$ ${ }^{224}$ in medium lacking (Control) or containing $0.2 \%$ arabinose (Induced).

https://doi.org/10.1371/journal.pgen.1009550.g005 
WT VqmA $A_{V c}$, eliminating the possibility that the observed binding to Pqtip was a consequence of overexpression (S7 Fig). We conclude that the region encompassing both the HTH motif and the C-terminal 25 residues are required to restrict the $\mathrm{VqmA}_{\mathrm{Vc}} \mathrm{DBD}$ from binding Pqtip.

\section{Discussion}

The DPO-VqmA QS AI-receptor pair controls lifestyle transitions in the pathogen $V$. cholerae and in the vibriophage VP882. Here, we studied the DNA-binding function of VqmA. VqmA proteins are cytoplasmic transcription factors composed of $\mathrm{N}$-terminal PAS domains responsible for binding the DPO ligand and C-terminal DBDs containing HTH motifs. Most of the key residues required for binding the DPO ligand and for binding to P $v q m R$ DNA are conserved between the two VqmA proteins. Indeed, both $\mathrm{VqmA}_{\mathrm{Vc}}$ and $\mathrm{VqmA}_{\text {Phage }}$ bind $\mathrm{DPO}$ and activate transcription of $v q m R$. By contrast, only $\mathrm{VqmA}_{\text {Phage }}$ activates the phage gene qtip. Here, we investigated this asymmetric DNA-binding pattern. Our work shows that, in both proteins, the DBD determines promoter recognition. We have previously shown that DPO binding enhances VqmA transcriptional activity [9]. This earlier work, together with our present results, suggest a model in which the PAS domain specifies DNA-binding affinity (between the apo- and holo- states), and the DBD specifies DNA-binding selectivity.

The main goal of the present work was to discover features of the VqmA proteins that confer specificity in transcriptional activity. We propose that phage VP882 $\mathrm{VqmA}_{\text {Phage }}$ possesses a feature that relaxes its DNA-binding specificity and $V$. cholerae $\mathrm{VqmA}_{\mathrm{Vc}}$ possesses a feature that restricts its DNA-binding capability. Regarding $\mathrm{VqmA}_{\mathrm{Vc}}$, our genetic analyses support the hypothesis that the $\mathrm{VqmA}_{\mathrm{Vc}} \mathrm{DBD}$ harbors elements that prevent it from binding Pqtip. This hypothesis stems from our finding that residues G201, A202, E207, and M211 are crucial for $\mathrm{VqmA}_{\text {Phage }}$ recognition of Pqtip. These residues are conserved between $\mathrm{VqmA}_{\mathrm{Vc}}$ and $\mathrm{VqmA}_{\text {Phage. }}$ Specifically, in $\mathrm{VqmA}_{\mathrm{Vc}}$ they are: G199, A200, Q205, and L209, respectively. More broadly, sequence alignments of VqmA proteins among Vibrios reveal that the residue at the $207^{\text {th }}$ position in $\mathrm{VqmA}_{\text {Phage }}\left(205^{\text {th }}\right.$ position in $\left.\mathrm{VqmA}_{\mathrm{Vc}}\right)$ is most frequently either a Glu or a Gln [5]. Similarly, the residue at the $211^{\text {th }}$ position in $\mathrm{VqmA}_{\text {Phage }}\left(209^{\text {th }}\right.$ position in $\left.\mathrm{VqmA}_{\mathrm{Vc}}\right)$ is commonly a hydrophobic residue, like Met, Leu, Ile, or Val. Thus, E207 and M211 are not unique to $\mathrm{VqmA}_{\text {Phage }}$, but rather occur in most $\mathrm{VqmA}$ proteins. We propose that because the key residues for Pqtip binding are conserved in $\mathrm{VqmA}_{\text {Phage, }}, \mathrm{VqmA}_{\mathrm{Vc}}$, and other Vibrio VqmA proteins, $\mathrm{VqmA}_{\mathrm{Vc}}$ is likely restricted from binding Pqtip by additional features elsewhere in its DBD. Regarding $\mathrm{VqmA}_{\text {Phage }}$, the $\mathrm{DPO}-\mathrm{VqmA}_{\text {Phage }}$ structure was reported during review of this manuscript [15]. Superimposition of this new structure (7DWM) onto the DPO-VqmA $A_{V c}$ and $\mathrm{DPO}-\mathrm{VqmA}_{\mathrm{Vc}_{\mathrm{c}}} \mathrm{P} v q m R$ structures $(6 \mathrm{KJU}$ and $6 \mathrm{IDE}$, respectively, and $[9,11,14])$ reveals two insights (S8 Fig). First, the conformations of the three PAS domains are similar except for the orientations of the first $20 \mathrm{~N}$-terminal residues in each protein, indicating that the PAS domains do not confer the differences in promoter DNA specificity. Second, the DPO$\mathrm{VqmA}_{\text {Phage }} \mathrm{DBDs}$ adopt a conformation that is intermediate between that of the more open DBDs in the DPO- $\mathrm{VqmA}_{\mathrm{Vc}}$ structure and the closed DBDs in the DPO-VqmA $\mathrm{Vc}_{\mathrm{Vc}}-\mathrm{P} v q m R$ structure. Additionally, the interaction interface between the $\mathrm{VqmA}_{\text {Phage }} \mathrm{DBDs}$ is less extensive, and thus more relaxed than that of the $\mathrm{VqmA}_{\mathrm{Vc}} \mathrm{DBDs}$ [15]. Likely, the more relaxed conformation exhibited by the VqmA $A_{\text {Phage }}$ DBDs underpins its promiscuity for promoter binding with respect to $\mathrm{P} v q m R$ and $\mathrm{P} q$ tip.

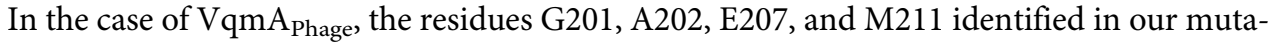
genesis screen as necessary for Pqtip binding are, surprisingly, not in the HTH motif, nor do the corresponding $\mathrm{VqmA}_{\mathrm{Vc}}$ residues make direct contacts with DNA in the DPO-VqmA $\mathrm{Vc}^{-}$ P vqmR crystal structure (Fig 4D). Thus, we wonder how the G201, A202, E207, and M211 
residues could govern recognition of Pqtip. Our in vivo analyses showed that substitutions in $\mathrm{VqmA}_{\text {Phage }}$ at these residues enable activation of $v q m R$ expression to WT levels, whereas only residual activation of qtip expression occurs (Fig 4A-4C). Surprisingly, the purified $\mathrm{VqmA}_{\text {Phage }}$ mutant proteins maintained some capability to bind Pqtip in vitro. A representative experiment using the $\mathrm{VqmA}_{\text {Phage }}{ }^{\mathrm{G} 201 \mathrm{D}}$ protein is shown in S9A Fig.

We consider several possibilities to explain our findings:

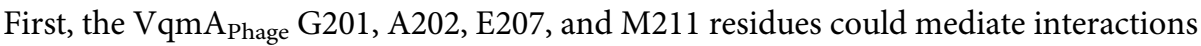
with an additional bacterial factor involved in transcription. Importantly, the failure of these $V_{\text {VqmA }} A_{\text {Phage }}$ variants to activate Pqtip expression in V. cholerae lysogens also occurred in E. coli, eliminating the possibility that these residues interact with a phage-specific or Vibrio-specific factor. Rather, these residues could be important for coordinating interactions with a conserved factor, such as RNA polymerase. If so, these mutant $\mathrm{VqmA}_{\text {Phage }}$ proteins, while capable of binding promoter DNA, are incapable of activating transcription. This situation would be analogous to the positive control mutants of the lambda phage $\mathrm{cI}$ repressor $\left(\mathrm{cI}_{\mathrm{lambda}}\right)$. So called pc mutants bind DNA and exhibit repressor activity, but are deficient in positive transcriptional regulation due to the inability of the mutant $\mathrm{cI}_{\text {lambda }}$ proteins to productively interact with RNA polymerase $[16,17]$. In our case, the $\mathrm{VqmA}_{\text {Phage }}$ mutants maintain the capacity to activate $v q m R$ expression so they must successfully interact with RNA polymerase at least at $\mathrm{P} v q m R$. For this reason, we consider it unlikely that these $\mathrm{VqmA}_{\text {Phage }}$ mutants are analogous to lambda pc mutants.

Second, a global transcriptional regulator could be involved that is present in both $V$. cholerae and E. coli. One candidate is the histone-like nucleoid structuring protein (H-NS) that functions as a universal repressor of transcription [18]. In Vibrio harveyi, the QS master regulator, LuxR, displaces H-NS at promoter DNA to activate expression of QS-controlled genes [19]. Perhaps, the VqmA $A_{\text {Phage }}$ G201, A202, E207, and M211 mutants cannot successfully compete with H-NS for binding at Pqtip in vivo, whereas in an EMSA assay, since H-NS is not present, binding to Pqtip DNA occurs. To address this possibility, we examined whether WT $\mathrm{VqmA}_{\text {Phage }}$ and $\mathrm{VqmA}_{\text {Phage }}$ G201D competed with H-NS for binding to Pqtip using EMSA assays. There was no difference between WT VqmA $A_{\text {Phage }}$ and VqmA $A_{\text {Phage }}{ }^{G 201 D}$ binding to Pqtip DNA in the presence of purified H-NS (S9C and S9D Fig). These experiments suggest that it is unlikely that $\mathrm{H}-\mathrm{NS}$ competition underlies our findings.

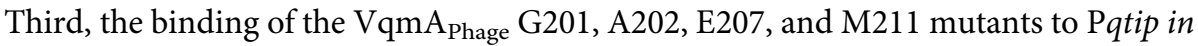
vitro, while demonstrating loss of activity in vivo, could be a consequence of the unnaturally high DNA: VqmA $A_{\text {Phage }}$ stoichiometry in the EMSA, similar to what we observed for the GST-DBD $_{\mathrm{Vc}_{\mathrm{c}}}$ construct (Fig $1 \mathrm{G}$ ). Thus, the EMSA is not sufficiently sensitive to distinguish

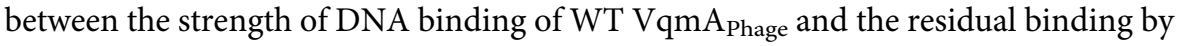
the VqmA $A_{\text {Phage }}$ G201, A202, E207, and M211 mutants. If this is the case, we propose that $\mathrm{VqmA}_{\text {Phage }}$ G201, A202, E207, and M211 could play allosteric roles in correctly positioning the VqmA $A_{\text {Phage }}$ HTH for proper contact with particular DNA nucleotides. Here, we compare this possibility to how site-specific recognition is accomplished by $\mathrm{cI}_{\text {lambda. }}$. Genetic and biochemical studies revealed that residues outside of the $\mathrm{cI}_{\text {lambda }} \mathrm{HTH}$ motif are crucial for sitespecific DNA recognition [20-24]. The crystal structure of the $\mathrm{cI}_{\text {lambda }}$ repressor bound to DNA shows that charged residues adjacent to those in the HTH interact with the DNA sugar phosphate backbone [25]. Additionally, the $\mathrm{N}$-terminal arm of $\mathrm{cI}_{\text {lambda }}$ wraps around the DNA and makes contacts on the backside of the helix [25]. It is presumed that the backbone contacts function to position the $\mathrm{HTH}$ residues to contact specific DNA nucleotides. Thus, while the VqmA $A_{\text {Phage }}$ residues that we identified as important for Pqtip recognition (G201, A202, E207, and M211) do not function perfectly analogously to those in $\mathrm{cI}_{\text {lambda }}$ because they do not make contact with the DNA backbone, their role in site-specific recognition could be similar. A 
caveat of our interpretation is that, as noted, we do not have a structure of $\mathrm{VqmA}_{\text {Phage }}$ bound to $\mathrm{P} q$ tip and we mapped the residues identified in our $\mathrm{VqmA}_{\mathrm{Phage}}$ mutagenesis to the $\mathrm{DPO}-\mathrm{VqmA} \mathrm{Vc}_{\mathrm{vc}} \mathrm{P} v q m R$ crystal structure. Therefore, it remains possible that the residues we identified here do indeed make contacts with DNA. A further possibility is that the residues we identified foster increased plasticity to the $\mathrm{VqmA}_{\text {Phage }} \mathrm{DBDs}$, perhaps, allowing $\mathrm{VqmA}_{\text {Phage }}$ to bind the longer palindrome that exists in Pqtip, which we discuss below. The recently reported DPO-VqmA $A_{\text {Phage }}$ crystal structure [15], together with the existing DPO-VqmA $A_{V c}$ structures, could enable modeling to predict the roles played by particular residues in conferring a relaxed conformation to the $\mathrm{VqmA}_{\text {Phage }} \mathrm{DBDs}$. To our knowledge, no region analogous to the one we discovered in $\mathrm{VqmA}_{\text {Phage }}$ has been shown to confer promoter specificity to a transcription factor. Going forward, determining the structure of $\mathrm{VqmA}_{\text {Phage }}$ bound to Pqtip DNA should reveal the mechanism enabling recognition of Pqtip and the role that these residues play, individually and collectively, in determining DNA-binding specificity.

Previous work demonstrated that $\mathrm{VqmA}_{\mathrm{Vc}}$ recognizes a key GG- $\mathrm{N}_{6}-\mathrm{CC}$ palindrome in $\mathrm{P} v q m R[2,11]$. Our sequence alignment of $\mathrm{P} v q m R$ and $\mathrm{P} q t i p$ showed that $\mathrm{P} q t i p$ does not possess this palindrome. Rather, the corresponding sequence in Pqtip is GG- $\mathrm{N}_{6}-\mathrm{TA}$ (Fig 2A). The most obvious divergence between the two sequences is in the central six nucleotides:

"-AAAATA-" in Pqtip and "-TTTCCC-" in PvqmR (Fig 2A). We hypothesized that this nucleotide stretch could be responsible for conferring the asymmetric DNA-binding patterns to the two VqmA proteins. Indeed, exchanging these nucleotides in Pqtip and $\mathrm{P} v q m R$ reversed the promoter binding preferences of the VqmA proteins. We verified our conclusion that this core 6 nucleotide stretch drives VqmA DNA-binding preference using our VqmA chimeric proteins $\left({ }_{\mathrm{Vc}} \mathrm{N}-\mathrm{C}_{\text {Phage }}\right.$ and $\left.{ }_{\text {Phage }} \mathrm{N}-\mathrm{C}_{\mathrm{Vc}}\right)$, a representative mosaic protein $\left(\mathrm{VqmA}_{\mathrm{Vc}}{ }^{* 171-224}\right)$, and a representative protein containing a point mutation $\left(\mathrm{VqmA}_{\text {Phage }}{ }^{\mathrm{G} 201 \mathrm{D}}\right)(\mathrm{S} 9 \mathrm{~A}, \mathrm{~S} 9 \mathrm{~B}$, and $\mathrm{S} 10$ Figs). While the present manuscript was under review, $\mathrm{Gu}$ et al. reported that a GG- $\mathrm{N}_{9}-\mathrm{CC}$ palindrome in Pqtip is the key sequence for VqmA $A_{\text {Phage }}$ recognition [15]. According to our DNA sequence alignment, the GG- $\mathrm{N}_{6}-\mathrm{CC}$ palindrome required for $\mathrm{VqmA}_{\mathrm{Vc}}$ binding is only present in $\mathrm{P} v q m R$, while the key GG- $\mathrm{N}_{9}$-CC palindrome required for $\mathrm{VqmA}_{\text {Phage }}$ binding exists in both Pqtip and $\mathrm{P} v q m R$ (Fig 2A). Together, our results and those of $\mathrm{Gu}$ et. al. [15] explain, at the level of the promoter DNA, why $\mathrm{VqmA}_{\text {Phage }}$ binds both Pqtip and $\mathrm{P} v q m R$ while $\mathrm{VqmA}_{\mathrm{Vc}}$ recognizes only $\mathrm{P} v q m R$.

Genomic sequencing data have revealed the presence of many QS receptor-transcription factors encoded in phage genomes [26]. In general, however, their transcriptional outputs are uncharacterized, with the exception of $\mathrm{VqmA}_{\text {Phage, }}$, which is promiscuous with respect to binding to $\mathrm{P} v q m R$ and $\mathrm{P} q t i p$, the only two promoters tested to our knowledge. It remains possible that $\mathrm{VqmA} \mathrm{A}_{\text {Phage }}$ regulates additional genes specifying bacterial and or/phage functions. Given that $\mathrm{VqmA}_{\text {Phage }}$ can regulate biofilm formation through its control of $V$. cholerae $v q m R$, probing the host regulon controlled by $\mathrm{VqmA}_{\text {Phage }}$ under various growth conditions could reveal unanticipated roles of QS in phage-Vibrio interactions.

Finally, we found that the $\mathrm{VqmA}_{\mathrm{V}_{c}}{ }^{\mathrm{A} 192 \mathrm{E}}$ variant exhibited modest, but detectable binding to $\mathrm{P} q$ tip, whereas the $\mathrm{VqmA}_{\mathrm{Vc}}$ quadruple mutant, and the $\mathrm{VqmA}_{\mathrm{Vc}}{ }^{* 171-199}$ mosaic protein did not. Western blot and $\mathrm{P} v q m R$-lux assays eliminated the possibility that any of the mutant proteins were not expressed or were misfolded. Rather, we infer that a particular regional conformation in the VqmA proteins is required for this key residue to function properly. Our results also show that exchanging both the $\mathrm{VqmA}_{\mathrm{Vc}} \mathrm{HTH}$ motif and C-terminal 25 residues with the corresponding residues from $\mathrm{VqmA}_{\text {Phage }}$ enables some but not WT-level binding to Pqtip. This finding supports the notion that a set of non-contiguous amino acids or a particular conformation of the $\mathrm{VqmA}_{\mathrm{Vc}} \mathrm{DBD}$ prevents binding to Pqtip. This arrangement is perhaps not surprising given that $V$. cholerae would pay a significant penalty if $\mathrm{VqmA}_{\mathrm{Vc}}$ bound the phage 
VP882 qtip promoter, as the consequence would be the launch of the phage lytic program and death of the host cell. To our knowledge, $\mathrm{VqmA}_{\mathrm{V}_{\mathrm{c}}}$ binds to only one promoter, $\mathrm{P} v q m R$ [3]. Thus, even in the context of the $V$. cholerae genome, $\mathrm{VqmA}_{\mathrm{Vc}_{\mathrm{c}}}$ transcriptional activity is tightly constrained. It is possible that other negative ramifications stem from non-specific $\mathrm{Vqm}_{\mathrm{Vc}}$ binding in the $V$. cholerae genome. Distinct mechanisms are employed to restrict other QS receptor/transcription factors from promiscuously binding to DNA. For example, LuxR-type QS receptors can typically bind $>100$ promoters, but their solubilization, stability, and DNAbinding capabilities strictly rely on being bound to an AI whose availability is, in turn, highly regulated [27-31]. Therefore, precise control of gene expression is maintained in many QS circuits by confining QS receptor activity to the ligand-bound form coupled with discrete affinities of the ligand-receptor complexes for target promoters. By contrast, $\mathrm{VqmA}_{\mathrm{Vc}}$ is expressed constitutively, and its DNA-binding capabilities are not limited by the presence of an AI. Thus, exquisitely tight control over promoter DNA-binding specificity by $\mathrm{VqmA}_{\mathrm{Vc}}$-restricting it to one and only one promoter-is apparently crucial for proper regulation of gene expression and survival.

\section{Materials and methods}

\section{Bacterial strains, plasmids, primers, and reagents}

Strains, plasmids, primers, and gBlocks used in this study are listed in S1-S4 Tables, respectively. In all experiments, $\Delta t d h V$. cholerae and $\Delta t d h$ E. coli strains were used except in the experiment assaying expression of $\mathrm{P} v q m R$-lux and $\mathrm{P} q$ tip-lux in response to the $\mathrm{DBD}_{\mathrm{Vc}}$,

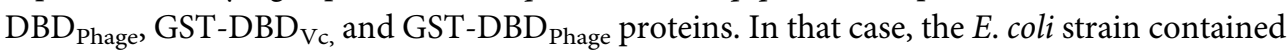
the WT $t d h$ gene. $V$. cholerae and E. coli were grown aerobically in lysogeny broth (LB) at $37^{\circ} \mathrm{C}$. Antibiotics and inducers were used at the following concentrations: 50 units $\mathrm{mL}^{-1}$ polymyxin $\mathrm{B}, 200 \mu \mathrm{g} \mathrm{mL}{ }^{-1}$ ampicillin, $5 \mu \mathrm{g} \mathrm{mL} \mathrm{L}^{-1}$ chloramphenicol, $100 \mu \mathrm{g} \mathrm{mL} \mathrm{L}^{-1}$ kanamycin, $0.2 \%$ arabinose, and $1 \mathrm{mM}$ Isopropyl $\beta$-D-1-thiogalactopyranoside (IPTG).

Primers were obtained from Integrated DNA Technologies. Gibson assembly, intramolecular reclosure, and traditional cloning methods were employed for all cloning. PCR with Q5 High Fidelity Polymerase (NEB) was used to generate insert and backbone DNA. Gibson assembly relied on HiFi DNA assembly mix (NEB). All enzymes used in cloning were obtained from NEB. Mutageneses of the $\mathrm{VqmA}_{\mathrm{Phage}}$ and $\mathrm{VqmA}_{\mathrm{Vc}} \mathrm{DBD}$ s were accomplished using the GeneMorph II EZClone Domain Mutagenesis Kit (Agilent) according to the manufacturer's instructions. Transfer of plasmids carrying vqmA genes into the $V$. cholerae phage VP882 lysogen employed conjugation followed by selective plating on polymyxin B, chloramphenicol, and kanamycin, based on previously described protocols [32].

\section{Genetic screens for $\mathrm{VqmA}_{\mathrm{Phage}}$ and $\mathrm{VqmA}_{\mathrm{Vc}}$ DNA-binding mutants}

E. coli carrying a library of plasmid-borne $v q m A_{\text {Phage }}$ mutants was mated with $V$. cholerae harboring a phage VP882 mutant ( $\left.v q m A_{\text {Phage: }}: T n 5\right)$ and the PvqmR-lux reporter integrated at the lacZ locus. Exconjugant $V$. cholerae colonies were collected and streaked onto LB agar plates supplemented with polymyxin B, chloramphenicol, kanamycin, and arabinose. $\mathrm{P} v q m R$-lux activity of surviving exconjugants was assayed using an ImageQuant LAS4000 imager (GE). $V$. cholerae colonies that produced light were harvested for plasmid DNA preparation. Isolated plasmid DNA was subsequently transformed into E. coli strains carrying Pqtip-lux or PvqmRlux to validate activity.

A library of plasmid-borne $v q m A_{V c}$ mutants was transformed into E. coli carrying the Pqtip-lux reporter. Transformants were plated on LB agar supplemented with ampicillin, 
kanamycin, and arabinose. Pqtip-lux activity was assayed using an ImageQuant LAS4000 imager.

\section{Growth, lysis, and bioluminescence assays}

To measure growth of V. cholerae phage VP882 lysogens or activation of the PvqmR-lux and Pqtip-lux reporters in bacterial strains, overnight cultures of $V$. cholerae or E. coli were backdiluted 1:1000 into LB medium supplemented with appropriate antibiotics prior to being dispensed $(200 \mu \mathrm{L})$ into 96-well plates (Corning Costar 3904). Arabinose was added as specified. The plates were shaken at $37^{\circ} \mathrm{C}$ and a Biotek Synergy Neo2 Multi-Mode reader was used to measure $\mathrm{OD}_{600}$ and bioluminescence. For bioluminescence assays, relative light units (RLU) were calculated by dividing bioluminescence by the $\mathrm{OD}_{600}$ after $5 \mathrm{~h}$.

\section{Protein expression, purification, and electrophoretic mobility shift assay (EMSA)}

Protein expression and purification were performed as described [9,19]. EMSAs were performed as described [8] with the following modifications: Following electrophoresis, 6\% DNA retardation gels were stained with SYBR Green (Thermo) and visualized using an ImageQuant LAS 4000 imager with the SYBR Green settings. Unless specified otherwise, the highest concentration of VqmA assessed was $600 \mathrm{nM} .25 \mathrm{nM}$ P vqmR or Pqtip DNA was used in all EMSAs. The percentage of promoter DNA bound was calculated using the gel analyzer tool in ImageJ and the estimated $\mathrm{EC}_{50}$ values were derived from $\mathrm{EC}_{50}$ analyses in Prism.

\section{Western blot analysis}

Western blot analyses probing for abundances of 3xFLAG-tagged proteins were performed as reported [3] with the following modifications: E. coli and V. cholerae carrying N-terminal 3xFLAG-tagged $\mathrm{VqmA}_{\mathrm{Vc}}$ and $\mathrm{N}$-terminal 3xFLAG-tagged $\mathrm{VqmA}_{\text {Phage }}$ alleles were backdiluted 1:1000 in LB supplemented with appropriate antibiotics and harvested after $6 \mathrm{~h}$ and $4 \mathrm{~h}$ of growth at $37^{\circ} \mathrm{C}$, respectively. Cells were resuspended in Laemmli sample buffer at a final concentration of $0.006 \mathrm{OD} / \mu \mathrm{L}$. Following denaturation for $15 \mathrm{~min}$ at $95^{\circ} \mathrm{C}, 5 \mu \mathrm{L}$ of each sample was subjected to SDS-PAGE gel electrophoresis. RpoA was used as the loading control (Biolegend Inc.). Signals were visualized using an ImageQuant LAS 4000 imager.

\section{Sequence alignments}

Protein and DNA sequences in FASTA format were aligned in the BioEdit Sequence Alignment Editor using the default setting under the ClustalW mode. Figs 2A and S1 were prepared via the ESPript 3.0 online server [33].

\section{Statistical methods}

All statistical analyses were performed using GraphPad Prism software. Error bars correspond to standard deviations of the means of three biological replicates.

\section{Supporting information}

S1 Fig. Sequence alignment of VqmA proteins. Protein sequence alignment (ClustalW) showing $\mathrm{VqmA}_{\mathrm{Vc}}$ and $\mathrm{VqmA}_{\text {Phage. }}$. Black and white boxes designate identical and conserved residues, respectively. The PAS domain and HTH motif are indicated. The site used to fuse domains for chimera constructions is indicated by the red box. Key residues required for DPO binding are designated with black triangles. Conserved HTH residues are designated by black 
circles and open circles show residues with different charges in the HTH motifs of the two proteins. The residue in each HTH motif that contributes to Pqtip specificity is designated by the striped circle. The residues identified in the $\mathrm{VqmA}_{\text {Phage }}$ screen and the equivalent residues altered by site-directed mutagenesis in $\mathrm{VqmA}_{\mathrm{Vc}}$ are designated by asterisks.

(TIF)

S2 Fig. VqmA $A_{\text {Phage }}$ has higher affinity for Pqtip DNA than for PvqmR DNA. (A) EC $\mathrm{E}_{50}$ analysis of the designated VqmA proteins for binding to P $v q m R$ and Pqtip. Data are representative of two independent experiments. The percentage of DNA bound was calculated using the gel analyzer tool in ImageJ and the estimated $\mathrm{EC}_{50}$ values were derived from Prism. (B) Competitive $\mathrm{VqmA}_{\text {Phage }}$ and ${ }_{\mathrm{Vc}} \mathrm{N}-\mathrm{C}_{\text {Phage }}$ EMSA analysis. $25 \mathrm{nM} \mathrm{P} v q m R$ and Pqtip DNA were used and no protein (designated -) or 2-fold serially-diluted protein was added to the lanes. The lowest and highest protein (dimer) concentrations are $4.7 \mathrm{nM}$ and $1200 \mathrm{nM}$, respectively. (TIF)

S3 Fig. The VqmA $A_{V_{c}}$ and Vqm $A_{P h a g e} D B D s$ are non-functional. (A and $B$ ) Normalized reporter activity from WT E. coli harboring (A) PvqmR-lux or (B) Pqtip-lux and arabinoseinducible $\mathrm{VqmA}_{\mathrm{Vc}}, \mathrm{DBD}_{\mathrm{Vc}}, \mathrm{VqmA}_{\text {Phage, }}$ and $\mathrm{DBD}_{\text {Phage. }}$ Black, no arabinose; white, $0.2 \%$ arabinose. Data are represented as mean \pm SD (error bars) with $n=3$ biological replicates. (C) EMSAs of $\mathrm{DBD}_{\mathrm{V}_{\mathrm{c}}}$ and $\mathrm{DBD}_{\text {Phage }}$ proteins binding to $\mathrm{P} v q m R$ and Pqtip. $25 \mathrm{nM} \mathrm{P} v q m R$ or Pqtip DNA was used in all EMSAs with no protein (designated -) or 2-fold serial dilutions of proteins. The lowest and highest protein (dimer) concentrations are $18.75 \mathrm{nM}$ and $600 \mathrm{nM}$, respectively. (D) Gel filtration chromatogram showing $\mathrm{UV}_{280}$ traces for the purification of (left) $\mathrm{VqmA}_{\mathrm{V}_{\mathrm{c}}}, \mathrm{DBD}_{\mathrm{Vc}}$, and $\mathrm{GST}-\mathrm{DBD}_{\mathrm{V}_{\mathrm{c}}}$ and (right) $\mathrm{VqmA}_{\text {Phage }}, \mathrm{DBD}_{\text {Phage, }}$, and

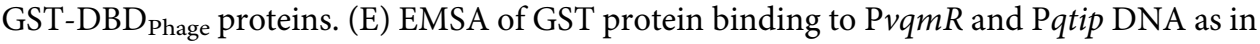
panel C.

S4 Fig. The VqmA $A_{P h a g e}$ and $V_{q m} A_{V c}$ variants are produced at levels similar to $W T$. West-

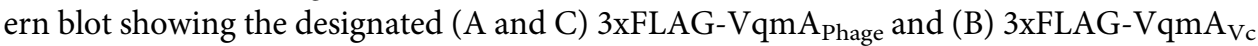
proteins produced by $\Delta t d h E$. coli and $\Delta t d h \Delta v q m A_{V c} V$. cholerae. A contaminating band below $\mathrm{VqmA}_{\text {Phage }}$ and $\mathrm{VqmA}_{\mathrm{Vc}}$ is present in all $\Delta t d h \mathrm{E}$. coli samples. The RNAP $\alpha$ subunit (RpoA) was used as the loading control. Data are representative of two independent experiments.

S5 Fig. Vqm $A_{\text {Phage }}$ mutants possessing WT activity induce phage lysis on agar plates supplemented with $0.2 \%$ arabinose. Shown is growth of $\Delta t d h \Delta v q m A_{V c} V$. cholerae harboring phage VP882 vqmA $A_{\text {Phage }}:$ Tn5 as a lysogen and arabinose-inducible $3 x$ FLAG-VqmA $A_{\text {Phage }}$ streaked onto agar plates with no arabinose (Control) or $0.2 \%$ arabinose (Induced). (TIF)

S6 Fig. VqmA ${ }_{V_{c}}{ }^{\text {Q205E }}$ and VqmA $_{\mathbf{V}_{c}}{ }^{\text {L209M }}$ do not bind Pqtip. (A and B) Normalized reporter activity from $\Delta t d h$ E. coli harboring (A) PvqmR-lux or (B) Pqtip-lux and arabinose-inducible $3 \times x_{L L A G}-\mathrm{VqmA}_{\mathrm{Vc}}, 3 \times \mathrm{xLAG}-\mathrm{VqmA}_{\text {Phage }}$, or the indicated $3 \mathrm{xFLAG} \mathrm{VqmA}_{\mathrm{Vc}}$ allele. Black, no arabinose; white, $0.2 \%$ arabinose. Data are represented as mean \pm SD (error bars) with $n=3$ biological replicates.

S7 Fig. VqmA $A_{V_{c}}$ mosaic proteins are produced at levels similar to $W T V q m A_{V c}$. Western blot showing the designated $3 \times \mathrm{FLAG}-\mathrm{VqmA} \mathrm{V}_{\mathrm{Vc}}$ mosaic proteins produced by $\Delta t d h \mathrm{E}$. coli and $\Delta t d h \Delta v q m A_{V c} V$. cholerae. RpoA was used as the loading control. Data are representative of 
two independent experiments.

(TIF)

S8 Fig. Structural comparisons of the $\mathrm{VqmA}_{\mathrm{Phage}}$ and $\mathrm{VqmA}_{\mathrm{Vc}}$ proteins. Previously reported crystal structures of DPO-VqmA $\mathrm{Vc}_{\mathrm{c}}-\mathrm{P} v q m R$ (blue, PDB: 6IDE) and DPO-VqmA $\mathrm{V}_{\mathrm{c}}$ (green, PDB: 6KJU) superimposed onto the recently published crystal structure of DPO-VqmA $A_{\text {Phage }}$ (yellow, PDB: 7DWM) based on the orientations of the PAS domains. DNA in the DPO-VqmA $\mathrm{Vc}_{\mathrm{c}} \mathrm{P} v q m R$ structure was omitted for simplicity. (TIF)

S9 Fig. EMSA analyses of the VqmA Phage $^{\text {G201D }}$ protein binding to DNA. (A) EMSA showing binding of VqmA Phage $^{\text {G201D }}$ to PvqmR and Pqtip DNA. 25 nM DNA was used in all EMSAs with no protein (designated -) or 2-fold serial dilutions of proteins. The lowest and highest protein (dimer) concentrations are $18.75 \mathrm{nM}$ and $600 \mathrm{nM}$, respectively. (B) As in panel A for PvqmR* and Pqtip* DNA. (C) EMSA showing WT VqmA Phage and VqmA ${ }_{\text {Phage }}{ }^{\text {G201D }}$ binding to Pqtip DNA in the presence of H-NS (300 nM). (D) EMSA showing H-NS binding to Pqtip DNA in the presence of WT VqmA Phage $_{\text {or VqmA }}$ G201D (each protein at $300 \mathrm{nM}$ ). (TIF)

S10 Fig. EMSA analyses of mosaic and chimeric VqmA proteins binding to $\mathrm{P} v q m R^{*}$ and Pqtip ${ }^{*}$ DNA. (A) EMSA showing binding of $\mathrm{VqmA}_{\mathrm{Vc}^{*}}{ }^{* 171-224}$ to P $v q m R$ and Pqtip DNA. 25 nM DNA was used in all EMSAs with no protein (designated -) or 2-fold serial dilutions of proteins. The lowest and highest protein (dimer) concentrations are $18.75 \mathrm{nM}$ and $600 \mathrm{nM}$, respectively. (B) As in panel A for PvqmR* and Pqtip* DNA. (C) As in panel A for ${ }_{\mathrm{Vc}} \mathrm{N}-\mathrm{C}_{\mathrm{Phage}}$ binding to $\mathrm{P} v q m R^{*}$ and Pqtip* DNA. (D) As in panel C for ${ }_{\mathrm{Phage}} \mathrm{N}-\mathrm{C}_{\mathrm{Vc}}$.

(TIF)

S1 Table. Bacterial strains used in this study.

(DOCX)

S2 Table. Plasmids used in this study.

(DOCX)

S3 Table. Primers used in this study.

(DOCX)

S4 Table. gBlocks used in this study. (DOCX)

S1 Data. Numerical data for Figs 1A, 1B, 1C, 1E, 1F, 2C, 2D, 3A, 3B, 3C, 3D, 3E, 3F, 4A, 4B, 4C, 5A, 5B, 5C, 5D, 5E, S2A, S3A, S3B, S3D, S6A and S6B. (XLSX)

\section{Acknowledgments}

We thank members of the Bassler laboratory for insightful discussions.

\section{Author Contributions}

Conceptualization: Olivia P. Duddy, Xiuliang Huang, Justin E. Silpe, Bonnie L. Bassler.

Data curation: Olivia P. Duddy, Xiuliang Huang, Justin E. Silpe.

Formal analysis: Olivia P. Duddy, Xiuliang Huang, Justin E. Silpe, Bonnie L. Bassler.

Funding acquisition: Bonnie L. Bassler. 
Investigation: Olivia P. Duddy, Xiuliang Huang, Justin E. Silpe, Bonnie L. Bassler.

Methodology: Olivia P. Duddy, Xiuliang Huang, Justin E. Silpe.

Project administration: Bonnie L. Bassler.

Resources: Olivia P. Duddy, Xiuliang Huang, Justin E. Silpe.

Supervision: Bonnie L. Bassler.

Validation: Olivia P. Duddy, Xiuliang Huang, Justin E. Silpe, Bonnie L. Bassler.

Visualization: Olivia P. Duddy, Xiuliang Huang, Justin E. Silpe.

Writing - original draft: Olivia P. Duddy, Xiuliang Huang, Justin E. Silpe, Bonnie L. Bassler.

Writing - review \& editing: Olivia P. Duddy, Xiuliang Huang, Justin E. Silpe, Bonnie L.

Bassler.

\section{References}

1. Papenfort K, Bassler BL. Quorum sensing signal-response systems in Gram-negative bacteria. Nat Rev Microbiol. 2016 Sep 1; 14(9):576-88. https://doi.org/10.1038/nrmicro.2016.89 PMID: 27510864

2. Papenfort K, Förstner KU, Cong JP, Sharma CM, Bassler BL. Differential RNA-seq of Vibrio cholerae identifies the VqmR small RNA as a regulator of biofilm formation. Proc Natl Acad Sci U S A. 2015 Feb 17; 112(7):E766-75. https://doi.org/10.1073/pnas.1500203112 PMID: 25646441

3. Papenfort K, Silpe JE, Schramma KR, Cong JP, Seyedsayamdost MR, Bassler BL. A Vibrio cholerae autoinducer-receptor pair that controls biofilm formation. Nat Chem Biol. 2017 May 1; 13(5):551-7. https://doi.org/10.1038/nchembio.2336 PMID: 28319101

4. Herzog R, Peschek N, Fröhlich KS, Schumacher K, Papenfort K. Three autoinducer molecules act in concert to control virulence gene expression in Vibrio cholerae. Nucleic Acids Res. 2019; 47(6):317183. https://doi.org/10.1093/nar/gky1320 PMID: 30649554

5. Silpe JE, Bassler BL. A host-produced quorum-sensing autoinducer controls a phage lysis-lysogeny decision. Cell. 2019 Jan 10; 176(1-2):268-280.e13. https://doi.org/10.1016/j.cell.2018.10.059 PMID: 30554875

6. Erez Z, Steinberger-Levy I, Shamir M, Doron S, Stokar-Avihail A, Peleg Y, et al. Communication between viruses guides lysis-lysogeny decisions. Nature. 2017 Jan 26; 541(7638):488-93. https://doi. org/10.1038/nature21049 PMID: 28099413

7. Silpe JE, Bassler BL. Phage-encoded LuxR-type receptors responsive to host-produced bacterial quorum-sensing autoinducers. MBio. 2019 Mar 1; 10(2):e00638-19. https://doi.org/10.1128/mBio.0063819 PMID: 30967469

8. Silpe JE, Bridges AA, Huang X, Coronado DR, Duddy OP, Bassler BL. Separating functions of the phage-encoded quorum-sensing-activated antirepressor Qtip. Cell Host Microbe. 2020 Feb 19; 27:629-641.e4. https://doi.org/10.1016/j.chom.2020.01.024 PMID: 32101705

9. Huang X, Duddy OP, Silpe JE, Paczkowski JE, Cong J, Henke BR, et al. Mechanism underlying autoinducer recognition in the Vibrio cholerae DPO-VqmA quorum-sensing pathway. J Biol Chem. 2020 Mar 6; 295(10):2916-31. https://doi.org/10.1074/jbc.RA119.012104 PMID: 31964715

10. Liu Z, Hsiao A, Joelsson A, Zhu J. The transcriptional regulator VqmA increases expression of the quorum-sensing activator HapR in Vibrio cholerae. J Bacteriol. 2006 Apr 1; 188(7):2446-53. https://doi.org/ 10.1128/JB.188.7.2446-2453.2006 PMID: 16547031

11. Wu H, Li M, Guo H, Zhou H, Li B, Xu Q, et al. Crystal structure of the Vibrio cholerae VqmA-ligandDNA complex provides insight into ligand-binding mechanisms relevant for drug design. J Biol Chem. 2019 Feb 22; 294(8):2580-92. https://doi.org/10.1074/jbc.RA118.006082 PMID: 30610119

12. Hayes JD, Pulford DJ. The glutathione s-transferase supergene family: Regulation of GST and the contribution of the Isoenzymes to cancer chemoprotection and drug resistance part i. Crit Rev Biochem Mol Biol. 1995; 30(6):445-520. https://doi.org/10.3109/10409239509083491 PMID: 8770536

13. Tudyka T, Skerra A. Glutathione S-transferase can be used as a C-terminal, enzymatically active dimerization module for a recombinant protease inhibitor, and functionally secreted into the periplasm of Escherichia coli. Protein Sci. 2008 Dec 31; 6(10):2180-7. 
14. Wu H, Li M, Peng C, Yin Y, Guo H, Wang W, et al. Large conformation shifts of Vibrio cholerae VqmA dimer in the absence of target DNA provide insight into DNA-binding mechanisms of LuxR-type receptors. Biochem Biophys Res Commun. 2019 Dec 3; 520(2):399-405. https://doi.org/10.1016/j.bbrc. 2019.10.063 PMID: 31606206

15. Gu Y, Zhi SX, Yang N, Yang WS. Understanding the mechanism of asymmetric gene regulation determined by the VqmA of vibriophage. Biochem Biophys Res Commun. 2021; 558:51-6. https://doi.org/ 10.1016/j.bbrc.2021.04.036 PMID: 33895551

16. Hochschild A, Irwin N, Ptashne M. Repressor structure and the mechanism of positive control. Cell. 1983 Feb 1; 32(2):319-25. https://doi.org/10.1016/0092-8674(83)90451-8 PMID: 6218886

17. Hawley DK, McClure WR. The effect of a lambda repressor mutation on the activation of transcription initiation from the lambda PRM promoter. Cell. 1983 Feb 1; 32(2):327-33. https://doi.org/10.1016/ 0092-8674(83)90452-x PMID: 6218887

18. Dorman CJ. H-NS: A universal regulator for a dynamic genome. Nat Rev Microbiol. 2004 May; 2 (5):391-400. https://doi.org/10.1038/nrmicro883 PMID: 15100692

19. Chaparian RR, Tran MLN, Miller Conrad LC, Rusch DB, Van Kessel JC. Global H-NS counter-silencing by LuxR activates quorum sensing gene expression. Nucleic Acids Res. 2020 Jan 10; 48(1):171-83. https://doi.org/10.1093/nar/gkz1089 PMID: 31745565

20. Pabo CO, Lewis M. The operator-binding domain of $\lambda$ repressor: Structure and DNA recognition. Nature. 1982; 298(5873):443-7. https://doi.org/10.1038/298443a0 PMID: 7088190

21. Hecht MH, Sauer RT. Phage lambda repressor revertants. Amino acid substitutions that restore activity to mutant proteins. J Mol Biol. 1985 Nov 5; 186(1):53-63. https://doi.org/10.1016/0022-2836(85)902566 PMID: 2934554

22. Lewis M, Jeffrey A, Wang J, Ladner R, Ptashne M, Pabo CO. Structure of the operator-binding domain of bacteriophage $\lambda$ repressor: Implications for DNA recognition and gene regulation. Cold Spring Harb Symp Quant Biol. 1982; 47(1):435-40.

23. Eliason JL, Weiss MA, Ptashne M. NH2-terminal arm of phage $\lambda$ repressor contributes energy and specificity to repressor binding and determines the effects of operator mutations. Proc Natl Acad Sci U S A. 1985 Apr 1; 82(8):2339-43. https://doi.org/10.1073/pnas.82.8.2339 PMID: 3157988

24. Nelson HCM, Sauer RT. Lambda repressor mutations that increase the affinity and specificity of operator binding. Cell. 1985 Sep 1; 42(2):549-58. https://doi.org/10.1016/0092-8674(85)90112-6 PMID: 3161621

25. Jordan SR, Pabo CO. Structure of the lambda complex at $2.5 \AA$ resolution: Details of the repressoroperator interactions. Science. 1988; 242(4880):893-9. https://doi.org/10.1126/science.3187530 PMID: 3187530

26. Duddy OP, Bassler BL. Quorum sensing across bacterial and viral domains. PLOS Pathog. 2021 Jan 7; 17(1):e1009074. https://doi.org/10.1371/journal.ppat.1009074 PMID: 33411743

27. van Kessel JC, Ulrich LE, Zhulin IB, Bassler BL. Analysis of activator and repressor functions reveals the requirements for transcriptional control by LuxR, the master regulator of quorum sensing in Vibrio harveyi. MBio. 2013 Jul 9; 4(4). https://doi.org/10.1128/mBio.00378-13 PMID: 23839217

28. Zhu J, Winans SC. Autoinducer binding by the quorum-sensing regulator TraR increases affinity for target promoters in vitro and decreases TraR turnover rates in whole cells. Proc Natl Acad Sci U S A. 1999 Apr 27; 96(9):4832-7. https://doi.org/10.1073/pnas.96.9.4832 PMID: 10220379

29. Swem LR, Swem DL, O'Loughlin CT, Gatmaitan R, Zhao B, Ulrich SM, et al. A quorum-sensing antagonist targets both membrane-bound and cytoplasmic receptors and controls bacterial pathogenicity. Mol Cell. 2009 Jul 31; 35(2):143-53. https://doi.org/10.1016/j.molcel.2009.05.029 PMID: 19647512

30. Schuster M, Urbanowski ML, Greenberg EP. Promoter specificity in Pseudomonas aeruginosa quorum sensing revealed by DNA binding of purified LasR. Proc Natl Acad Sci U S A. 2004 Nov 9; 101 (45):15833-9. https://doi.org/10.1073/pnas.0407229101 PMID: 15505212

31. Zhu J, Winans SC. The quorum-sensing transcriptional regulator TraR requires its cognate signaling ligand for protein folding, protease resistance, and dimerization. Proc Natl Acad Sci U S A. 2001 Feb 13; 98(4):1507-12. https://doi.org/10.1073/pnas.98.4.1507 PMID: 11171981

32. Bassler BL, Wright M, Showalter RE, Silverman MR. Intercellular signalling in Vibrio harveyi: sequence and function of genes regulating expression of luminescence. Mol Microbiol. 1993; 9(4):773-86. https:// doi.org/10.1111/j.1365-2958.1993.tb01737.x PMID: 8231809

33. Robert $X$, Gouet $P$. Deciphering key features in protein structures with the new ENDscript server Nucleic Acids Res. 2014 Jul 1; 42(W1):W320-4. https://doi.org/10.1093/nar/gku316 PMID: 24753421 